\title{
Effect of Intervention of Vaccination and Treatment on the Transmission Dynamics of HBV Disease: A Mathematical Model Analysis
}

\author{
Firaol Asfaw Wodajo $(i)$ and Temesgen Tibebu Mekonnen \\ Department of Mathematics, Debre Berhan University, Debre Berhan, Ethiopia \\ Correspondence should be addressed to Firaol Asfaw Wodajo; fasfaw22@gmail.com
}

Received 9 October 2021; Accepted 27 December 2021; Published 21 January 2022

Academic Editor: Kolade M. Owolabi

Copyright (c) 2022 Firaol Asfaw Wodajo and Temesgen Tibebu Mekonnen. This is an open access article distributed under the Creative Commons Attribution License, which permits unrestricted use, distribution, and reproduction in any medium, provided the original work is properly cited.

Hepatitis B is a globally infectious disease. It is pretty contagious and can be transmitted by blood or bodily fluids, through things like sharing razors and toothbrushes. It has been called the silent killer because it is asymptomatic, one might have the virus but not know until it manifests itself until much later. Since people do not give attention, it will develop into cirrhosis and hepatocellular carcinoma that leads to liver transplantation and death. This nature of HBV disease motivated us to perform this work. Mathematical modeling of HBV transmission is an interesting research area. In this paper, we present characteristics of $\mathrm{HBV}$ virus transmission in the form of a mathematical model. We proposed and analyzed a compartmental nonlinear deterministic mathematical model SEACTR for transmission dynamics and control of hepatitis B virus disease. In this model, we used force infection which takes the contact rate of susceptible population and transmission probability into account. We proved that the solution of the considered dynamical system is positive and bounded. The model is studied qualitatively using the stability theory of differential equations and the effective reproductive number which represents the epidemic indicator is obtained from the largest eigenvalue of the next-generation matrix. Both local and global asymptotic stability conditions for disease-free and endemic equilibria are determined. The sensitivity index shows that the transfer rate from exposed class to acute infective class and transfer rate from exposed class to chronic infective class are the most dominant parameters contributing to the transmission of HBV. On the one hand, the vaccination rate and treatment rate are the parameters that suppress the transmission of the disease the most, and enhancing the vaccination rate for newborns and treatment for chronically infected individuals is very effective to stop the transmission of HBV. The combined efforts of vaccination, effective treatment, and interruption of transmission make elimination of the infection plausible and may eventually lead to the eradication of the virus.

\section{Introduction}

Hepatitis means inflammation of the liver. The liver is a vital organ that processes nutrients, filters blood, and fights infections [1]. When the liver is inflamed or damaged, its function can be affected. Heavy alcohol use, toxins, some medications, and certain medical conditions can cause hepatitis $[2,3]$. Hepatitis B is a potentially life-threatening liver infection caused by HBV and is a major global health problem and the most serious type of viral hepatitis (WHO, 2008). An estimated 600,000 persons die each year due to the acute or chronic consequences of hepatitis B (WHO, 2008; Shepard et al., 2006).

For researchers and policy advisors, mathematical modeling has proven to be a useful tool to those who compile and evaluate scientific evidence for health interventions. Developing a mathematical model helps to synthesize information from different sources into a consistent framework that allows an integrated analysis of complex problems $[4,5]$. Mathematical models are often used to simulate the impact of various interventions or public health strategies and to provide quantitative predictions of how interventions 
might affect population health in the future by researchers in public health, who provide advice to policymakers. Mathematical modeling has a long history and has become an important tool in decision-making for public health in the last two decades in the field of infectious disease control. Mathematical modeling helped the World Health Organization (WHO) outbreak response team and decision-makers in national outbreak response units with the interpretation of outbreak data during the early phase of the epidemic during the influenza pandemic of 2009 [6].

More recently, during the large outbreak of Ebola in West Africa, mathematical modelers estimated key parameters for outbreak control such as the impact of case isolation, contact tracing with quarantine, and sanitary funeral practices on the numbers of new infections $[7,8]$. When a vaccine against Ebola became available, mathematical modeling helped researchers and outbreak responders to design ring vaccination trials that could lead to successful testing of the vaccine despite a decreasing exposure risk during the declining epidemic phase $[9,10]$. This experience has led the WHO to publish a guidance document on the design of vaccine efficacy trials during public health emergencies [6]. Models are used to generate projections of population health given demographic changes, distributions, and trends of risk factors in a population and possible effects of intervention programs especially in the area of chronic diseases [11-14].

Mathematical models can be a useful tool in this approach which helps us to optimize the use of finite sources or simply to the goal (the incidence of infection) control measures more impressively. A hepatitis B mathematical model was used to develop a strategy for eliminating HBV in New Zealand [2]. Van Den Driessche and Watmough [15] proposed an agestructure model to predict the dynamics of HBV transmission and evaluate the long-term effectiveness of the vaccination programme in China. Zhao et al. [16] developed a model to explore the impact of vaccination and other controlling measures of HBV infection. Owolabi (2020) proposed "mathematical modeling of viral kinetics under immune control during primary HIV-1 infection" and showed that extending the target-cell-limited model, by implementing a saturation term for HIV-infected cell loss dependent upon infected cell levels, is able to reproduce the diverse observed viral kinetic patterns without the assumption of a delayed immune response. Their results suggest that the immune response may have a significant effect on the control of the virus during primary infection and may support experimental observations that an anti-HIV immune response is already functional during peak viremia [17].

Farman et al. (2020) proposed "numerical treatment of a nonlinear dynamical hepatitis-B model: an evolutionary approach" and implemented evolutionary computational technique and Padé approximation (EPA) for the treatment of nonlinear hepatitis-B model. They concluded that evolutionary computational technique and Padé approximation (EPA) help to reduce contamination levels rapidly without the need to supply step size [18].

Naik et al. (2021) proposed "modeling the transmission dynamics of COVID-19 pandemic in Caputo type fractional derivative" to study the transmission of COVID-19. They attempted to study the pattern and the trend of spread of this disease and prescribe a mathematical model which governs the COVID-19 pandemic using the Caputo type derivative. They obtained results showing that the applied numerical technique is computationally strong for modeling the COVID-19 pandemic [19].

Naik et al. (2020) proposed "chaotic dynamics of a fractional-order HIV-1 model involving AIDS-related cancer cells" which involves the interactions between cancer cells, healthy $\mathrm{CD} 4+\mathrm{T}$ lymphocytes, and virus-infected $\mathrm{CD} 4+\mathrm{T}$ lymphocytes leading to chaotic behavior. The results of their work show that the order of the fractional derivative has a significant effect on the dynamic process [20].

Many models developed concerning HBV did not consider both vertical and horizontal modes of transmission at once. In this paper, we study the dynamics of hepatitis B virus (HBV) infection under the administration of vaccination and treatment, where $\mathrm{HBV}$ infection is transmitted in two ways through vertical transmission and horizontal transmission. We also considered HBV disease-induced death rate, level of infectiousness of chronic infective population, and exposure rate. This work will contribute to understanding which infective class plays more roles in transmitting HBV disease and the collaboration of vaccination and treatment interventions is the best strategy to decrease transmission of the disease. While the horizontal transmission is reduced through the administration of vaccination to those susceptible individuals especially for the new born populations, the vertical transmission gets reduced through the administration of treatment to chronically infected individuals; therefore, the vaccine and the treatment play different roles in controlling HBV. Since HBV is an asymptomatic and silent killer, we are motivated to study factors contributing to its transmission and controlling strategies.

\section{Model Description and Formulation}

Conceptual ideas and quantitative results from mathematical models are at the core of many reports and documents produced at public health institutes containing advice for policymakers. Therefore, modeling often in combination with health economic assessments potentially has great influence on policy decisions. The analysts usually base their work on "scenario analysis" (i.e., a comparison of various possible intervention strategies in a systematic way) using "dynamic transmission models" [2, 21].

In this model, we consider a SEACTR epidemic model by dividing the total population into six time-dependent classes based upon the nature of HBV disease, namely, S, susceptible; E, exposed; A, acutely infected; C, chronically infected; $\mathrm{T}$, treated; and $\mathrm{R}$, recovered classes. To make the meaning of each compartment clear and easily understandable, we stated the biological meaning of each compartment below.

\section{Biological Meaning}

Susceptible: susceptible person is someone who is not vaccinated or otherwise immune or a person with a weakened immune system who has a way for the virus to 
enter the body. For an infection to occur, the virus must enter a susceptible person's body and invade tissues, multiply, and cause a reaction. They are individuals who can be infected but have not yet contracted HBV but may contract it if exposed to any mode of its transmission.

Exposed are individuals who became infected but have not yet become infectious.

Acute infective refers to microbe living inside a host for a limited period of time, typically less than six months. They are a number of individuals who have contracted the HBV and are active or capable of transmitting it.

Chronic infective is characterized by the continued presence of infectious virus following the primary infection and may include chronic or recurrent disease. They are a number of individuals who although apparently healthy themselves harbor infections that can be transmitted to others.

Treated class are individuals who get treated by lamivudine, tenofovir, and other treatments after they became chronically infected.

Recovered class are individuals who get successful treatment or recover by natural immunity. They are the number of individuals who are recovered after treatment and are immune to the disease.

In this model, the total population $N(t)$ is divided into six compartments depending on the epidemiological status of individuals: susceptible $S(t)$, exposed $E(t)$, acutely infected $A(t)$, chronically infected $C(t)$, treated $T(t)$, and recovered $R(t)$. Susceptible population increases by the coming in of the birth flux rate $\mu-\mu p_{1} C-\mu \theta R$, loss of immunity rate $u_{2}$ from recovered population, and decreases by force of infection entering into exposed population, proportions of susceptible move to recovered class by vaccination by the rate $\tau$. Exposed population increases by the coming in of force of infection and decreases by transfer rate from exposed class to acutely infected class by rate of $c_{1}$ and transfer rate from exposed class to chronic infective by rate of $c_{2}$. Acute infective class increases by the transfer rate from exposed class to acute infective class $c_{1}$ and decreases by rate of moving from acute class to chronic class $\delta_{1}$ and natural death rate $\mu$. Chronic infective class increases by the coming in of transfer rate from $E$ to $C$, rate of moving from $A$ to $C$ class; for vertical transmission, we assume that a proportion $p_{1}$ of newborns from infected class are infected and it is denoted by the term $\mu p_{1} C,\left(p_{1}<1\right)$, and decreases by treatment rate $\sigma$, and disease-induced death rate $d$. Treatment class increases by treatment rate $\sigma$ and decreases by recovery rate $\varphi$. A proportion $\theta$ of newborns from recovered class is immune and it is denoted by $\theta(<1)$. Recovered class increases by recovery rate $\varphi$, vaccination rate $\theta$ with proportion of $\mu$, and by proportions of susceptible move to recovered class by vaccination rate $\tau$, and since vaccination is not perfect, it decreases by loss of immunity rate $u_{2}$.

(i) $c_{1}$ is the rate by which exposed individuals are transferred to chronic HBV carrier

(ii) $c_{2}$ is the rate by which exposed individuals are transferred to acute infective individuals (iii) $\delta_{1}$ is the rate at which individuals leave the acute infective class and enter chronic HBV carriers, $d$ is HBV disease-induced death rate

(iv) $\sigma$ is the rate of chronic infective individuals going for treatment

(v) $\delta_{2}$ is the rate by which acute infective individuals recover from the disease by natural way (spontaneous recovery)

(vi) The horizontal transmission of disease propagation is denoted by the mass action term $c \omega(A+\gamma C) S$, where $c$ is per capita contact rate, $\omega$ is probability of acquiring HBV infection per contact with one infectious individual, and $\gamma$ is level of infectiousness of chronic infective population

(vii) For vertical transmission, we assume that a proportion $p_{1}$ of newborns from infected class is infected and it is denoted by the term $\mu p_{1} C$, $\left(p_{1}<1\right)[22]$

(viii) Each compartment decreases by natural death rate $\mu$

We assume that the population of newborn carriers born to carriers is less than the sum of the death carriers and the population moving from carrier to recovery state. In this case, we have $\mu p_{1}<\mu+d+\sigma$; otherwise, carriers would keep increasing rapidly as long as there is infection; that is, $d C / \mathrm{dt}>0$ for $C \neq 0$ or $A \neq 0$.

Based up on the above assumptions, we constructed the corresponding flow chart shown in Figure 1

Based up on the flow diagram in Figure 1, we constructed the following corresponding dynamical system.

Corresponding dynamical system

$$
\begin{aligned}
& \dot{S}= \mu-\mu p_{1} C-\mu \theta R+u_{2} R \\
&-(c \omega(A+\gamma C)+\mu+\tau) S, \\
& \dot{E}=c \omega(A+\gamma C) S-\left(c_{1}+c_{2}+\mu\right) E, \\
& \dot{A}=c_{1} E-\left(\delta_{1}+\delta_{2}+\mu\right) A, \\
& \dot{C}=c_{2} E+\delta_{1} A-\left(\sigma+d+\mu-\mu p_{1}\right) C, \\
& \dot{T}=\sigma C-(\varphi+\mu) T, \\
& \dot{R}=\varphi T+\delta_{2} A+\tau S+\left(\mu \theta-\mu-u_{2}\right) R,
\end{aligned}
$$

\section{Mathematical Analysis of the Model}

In this section, the positivity, boundedness, and existence of the solution of the model are checked. This mathematical analysis of the model could be considered as the primary result.

Theorem 1 (positivity).

Let the initial data for the model be $S_{0}>0, E_{0}>0, A_{0}>0, C_{0}>0, T_{0}>0, R_{0}>0$. Then, the 


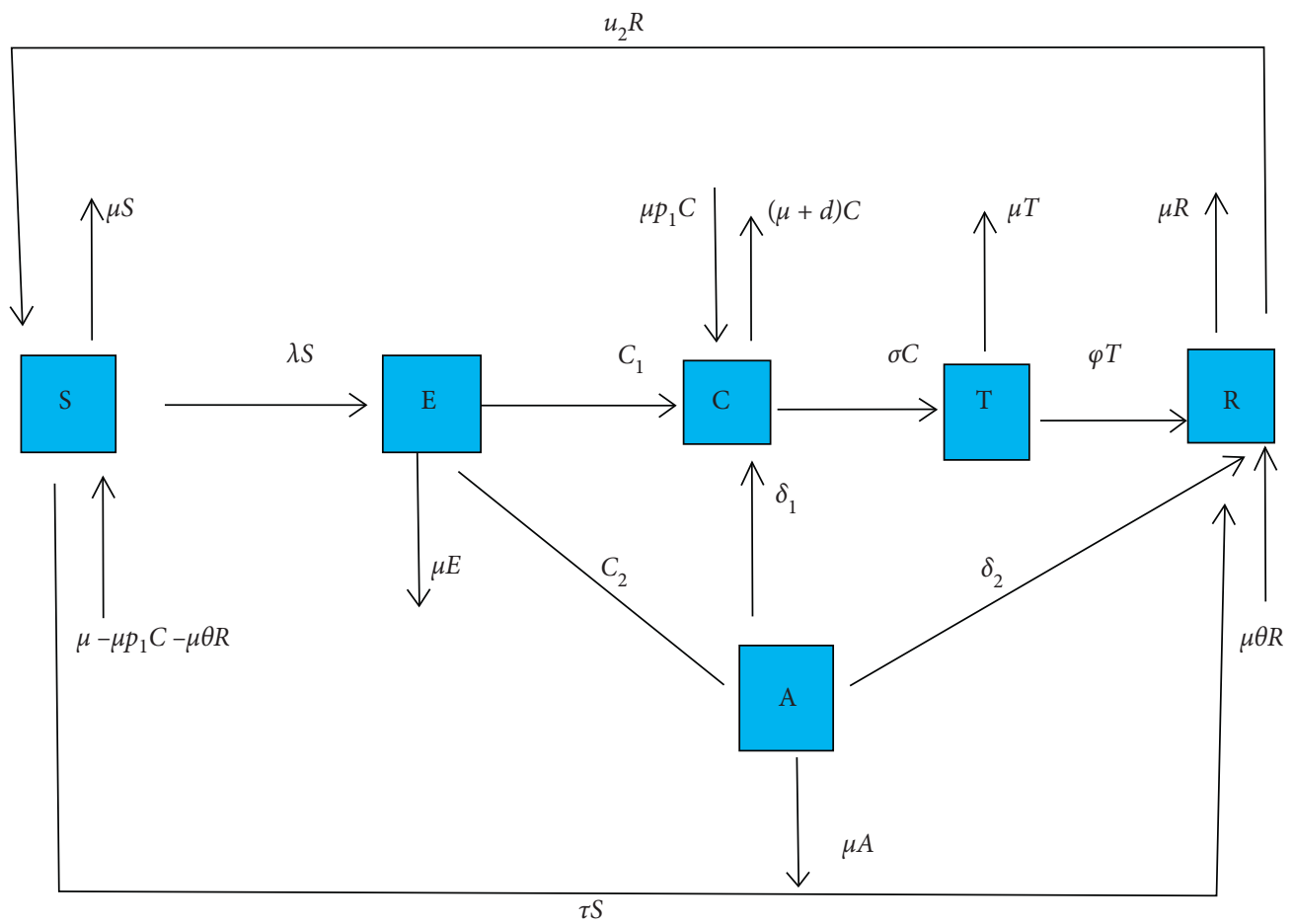

FIgURE 1: Corresponding flow diagram of SEACTR model.

solutions $S(t), E(t), A(t), C(t), T(t)$, and $R(t)$ of the model will be remaining positive for all time $t>0$.

Proof. Let $S_{0}>0, E_{0}>0, A_{0}>0, C_{0}>0, T_{0}>0, R_{0}>0$. Moreover, we assume that all parameters are positive. To show this, we take these differential equations of the dynamical system given above and show that their solutions are nonnegative as follows.

(1) Let us take the first differential equation

$$
\frac{d S}{d t}=\mu-\mu p_{1} C-\mu \theta R+u_{2} R-(c \omega(I+\gamma C)+\mu+\tau) S .
$$

After solving using the technique of separation of variables and applying the initial conditions, the following is obtained:

$\mathrm{S}(t) \geq S_{0} e^{-(\rho(I+\theta C)+\mu+\tau) t}$. Since $\quad S_{0}>0 \quad$ and $e^{-(c \omega(I+\gamma C)+\mu+\tau) t}$ is also positive, then we can conclude that

$$
\Rightarrow S(t) \geq S_{0} e^{-(c \omega(I+\gamma C)+\mu+\tau) t}>0 .
$$

(2) Let us consider the second ordinary differential equation

$$
\frac{d E}{d t}=c \omega(I+\gamma C) S-\left(c_{1}+c_{2}+\mu\right) E .
$$

After solving using the technique of separation of variable and applying the initial condition, the following is obtained:
$E(t) \geq E_{0} e^{-\left(c_{1}+c_{2}+\mu\right) t}$. Since $E_{0}$ is positive and $e^{-\left(c_{1}+c_{2}+\mu\right) t}$ is also positive, then we can conclude that

$$
\Rightarrow E(t) \geq E_{0} e^{-\left(c_{1}+c_{2}+\mu\right) t}>0
$$

(3) Let us consider the third ordinary differential equation

$$
\frac{d A}{d t}=c_{1} E-\left(\delta_{1}+\delta_{2}+\mu\right) A
$$

It is true that after solving using the technique of separation of variable and applying the initial condition, the following is obtained:

$\Rightarrow A(t) \geq A_{0} e^{-\left(\delta_{1}+\delta_{2}+\mu\right) t}$. Since $A_{0}$ is positive and $e^{-\left(\delta_{1}+\delta_{2}+\mu\right) t}$ is also positive, we can conclude that

$$
A(t) \geq A_{0} e^{-\left(\delta_{1}+\delta_{2}+\mu\right) t}>0 .
$$

(4) Let us consider the fourth differential equation

$$
\frac{d C}{d t}=c_{1} E+\delta_{1} A-\left(\sigma+d+\mu-\mu p_{1}\right) C .
$$

After solving using the technique of separation of variable and applying the initial condition, the following is obtained:

$C(t) \geq C_{0} e^{-\left(\sigma+d+\mu-\mu p_{1}\right) t}$. Since $A_{0}$ is positive and $e^{-\left(\sigma+d+\mu-\mu p_{1}\right) t}$ is also positive, then

$$
\Rightarrow C(t) \geq C_{0} e^{-\left(\sigma+d+\mu-\mu p_{1}\right) t}>0 .
$$


(5) Let us consider the fifth ordinary differential equation

$$
\frac{d T}{d t}=\sigma C-(\varphi+\mu) T .
$$

After solving using the technique of separation of variable and applying the initial condition, the following is obtained:

$T(t) \geq T_{0} e^{-(\varphi+\mu) t}$. Since $C_{0}$ is positive and $e^{-(\varphi+\mu) t}$ is also positive, then we can conclude that

$$
T(t) \geq T_{0} e^{-(\varphi+\mu) t}>0 .
$$

(6) Let us consider the sixth ordinary differential equation

$$
\frac{d R}{d t}=\varphi T+\delta_{2} A+\tau S+\left(\mu \theta-\mu-u_{2}\right) R .
$$

After solving using the technique of separation of variable and applying the initial condition, the following is obtained:
$R(t)=R_{0} e^{\left(\mu \theta-\mu-u_{2}\right) t}$. Since $R_{0}$ is positive and $e^{\left(\mu \theta-\mu-u_{2}\right) t}$ is also positive, then we can conclude that $R(t)=R_{0} e^{\left(\mu \theta-\mu-u_{2}\right) t}>0$ where $\mu \theta<\mu+\sigma$.

This completes the proof of the theorem. Therefore, the solution of the model is positive.

Theorem 2 (boundedness).

To show the boundedness of the solution, we have to show a lower bound and upper bound. But, initially, $N(0)=$ $N_{0}>0, S(0)=S_{0}>0, V(0)=\quad V_{0}>0, E(0)=E_{0}>0$, $A(0)=A_{0}>0, C(0)=C_{0}>0, T(0)=T_{0}>0, R(0)=R_{0}>0$.

These initial conditions are considered as lower bounds. Now, we are going to show the upper bound. By taking the relation,

$$
\begin{aligned}
N(t) & =S(t)+E(t)+A(t)+C(t)+T(t)+R(t), \\
& \Rightarrow R=1-S-E-A-C-T .
\end{aligned}
$$

Then, our system of differential equation becomes

$$
\begin{aligned}
& \dot{S}=\mu-\mu p_{1} C-(c \omega(A+\gamma C)+\mu+\tau) S+\left(u_{2}-\mu \theta\right)(1-S-E-A-C-T), \\
& \dot{E}=c \omega(A+\gamma C) S-\left(c_{1}+c_{2}+\mu\right) E, \\
& \dot{A}=c_{1} E-\left(\delta_{1}+\delta_{2}+\mu\right) A, \\
& \dot{C}=c_{2} E+\delta_{1} A-\left(\sigma+d+\mu-\mu p_{1}\right) C, \\
& \dot{T}=\sigma C-(\varphi+\mu) T .
\end{aligned}
$$

After substituting $R=1-S-E-A-C-T$ in the first equation, let $Y=S+E+A+C+T$. This implies

$$
\frac{d Y}{d t}=\frac{d S}{d t}+\frac{d E}{d t}+\frac{d A}{d t}+\frac{d C}{d t}+\frac{d T}{d t}
$$

After simplification, we get

$$
\frac{d Y}{d t}+\left(\mu+u_{2}-\mu \theta\right) Y \leq \mu+u_{2} .
$$

Now integrating both sides of the above inequality and using the theory of differential inequality $[3,28]$, we get the following:

This equation is in the form of $\mathrm{d} y / \mathrm{d} t+p(x) y=q(x)$ which is first-order linear differential equation with integrating factor $I . F=e^{\int-\left(\mu+u_{2}-\mu \theta\right) \mathrm{d} t}=e^{-\left(\mu+u_{2}-\mu \theta\right) t}$.

After solving and simplifying, we get $Y(t) \leq e^{-\left(\mu+u_{2}-\mu \theta\right) t}\left[\left(\left(\mu+u_{2}\right) e^{\left(\mu+u_{2}-\mu \theta\right) t} / \mu+u_{2}-\mu \theta\right)+k\right]$ where $k$ is constant, and letting $t \longrightarrow \infty$, we have $S+E+A+C+T \leq\left(\mu+u_{2}\right) /\left(\mu+u_{2}-\mu \theta\right)$.

From the first equation, we have $\dot{S}=\mu-\mu p_{1} C-(c \omega(A+\gamma C)+\mu+\tau) S+(\sigma-\mu \theta) \quad(1-S$ $-E-A-C-T)$. Then, $S \leq\left(\left(\mu+u_{2}\right) / \quad(\mu+\tau+\right.$ $\left.\left.u_{2}\right)-\mu \theta\right) . \Omega=\left\{(S, E, A, C, T) \in \quad R_{+}^{5} \mid S \leq\left(\mu+u_{2}\right) /(\mu+\tau\right.$ $\left.\left.+u_{2}-\mu \theta\right), S+E+A+C+T \leq\left(\mu+u_{2}\right) /\left(\mu+\tau+u_{2}-\mu \theta\right).\right\}$ is positively invariant.
Therefore, the basic model is well-posed epidemiologically and mathematically. Hence, it is sufficient to study the dynamics of the basic model in $\Omega$.

4.1. Disease Free Equilibrium Point $\left(E^{1}\right)$. To find disease free equilibrium point of (1)-(5), we use $E=A=C=0$ and equate each system of differential equation to zero. Then, we get

$$
E^{1}=\left(S^{0}, E^{0}, A^{0}, C^{0}, T^{0}, R^{0}\right)=\left(\frac{\mu-\mu \theta+\sigma}{\tau+\mu+\sigma-\mu \theta}, 0,0,0,0\right) .
$$

4.2. Effective Reproduction Number $\left(R_{\mathrm{Eff}}\right)$. The larger the number of people who are immune in a population, the lower the likelihood that a susceptible person will come into contact with the infection. It is more difficult for diseases to spread between individuals if large numbers are already immune as the chain of infection is broken.

The effective reproduction number $\left(R_{\mathrm{Eff}}\right)$ is used to measure the transmission potential of a disease. It is the average number of secondary infections produced by a typical case of an infection in a population where everyone is 
susceptible. The key threshold result of epidemic theory associates the outbreaks of epidemics and the persistence of endemic levels with effective reproduction numbers greater than one. Because the magnitude of $R_{\mathrm{Eff}}$ allows one to determine the amount of effort which is necessary either to prevent an epidemic or to eliminate an infection from a population, it is crucial to estimate $R_{\mathrm{Eff}}$ for a given disease in a particular population. We calculate the effective reproduction number denoted by $R_{\text {Eff }}$ using the Van Den Driessche and Watmouth next-generation matrix approach from $[15,29]$.

The effective reproduction number is obtained by taking the largest (dominant) eigenvalue (spectral radius) of the matrix: $F V^{-1}=\left[\partial \mathscr{F}_{i}\left(E^{1} / \partial x_{j}\right)\right]\left[\partial v_{i}\left(E^{1} / \partial x_{j}\right)\right]^{-1}$, where $\mathscr{F}_{i}$ is the rate of appearance of new infection in compartment $i, v_{i}$ is the transfer of infections from one compartment $i$ to another, and $E^{1}$ is the disease-free equilibrium point. The effective reproduction number $R_{\mathrm{Eff}}$ of our $\mathrm{HBV}$ model is obtained by rearranging the differential equation of the dynamical system (1)-(6) in terms of $\mathrm{d} X_{i} / \mathrm{d} t=\mathscr{F}_{i}-v_{i}=\mathscr{F}_{i}-\left(v_{i}^{-}-v_{i}^{+}\right)$. We find the effective reproduction number by using the next-generation matrix method such that the reproduction number is the dominant eigenvalue of the next-generation matrix $G=F V^{-1}$ where $F$ represents new infection and $V$ represents transfer of infections from one compartment to another $[15,29]$.

$F\left(E^{0}\right)=\left[\begin{array}{ccc}0 & c \omega S^{0} & c \omega \gamma S^{0} \\ 0 & 0 & 0 \\ 0 & 0 & 0\end{array}\right]$,

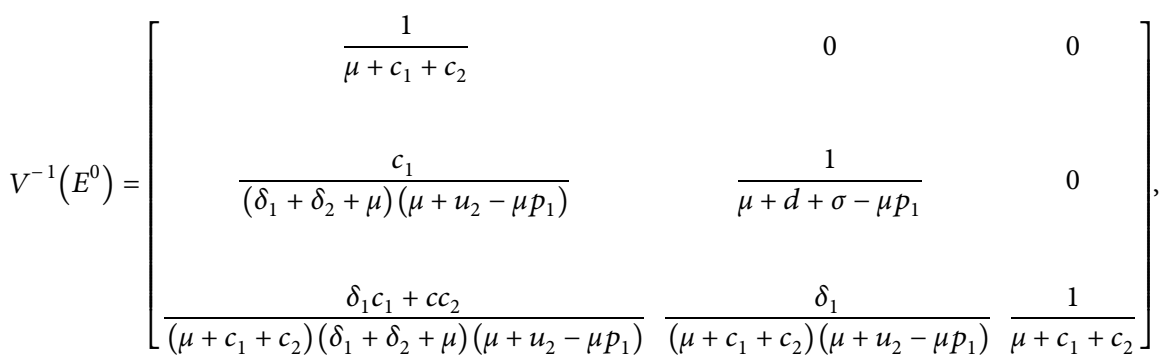

$F V^{-1}=\left[\begin{array}{cc}\frac{c \omega c_{1} S^{0}}{\left(\delta_{1}+\delta_{2}+\mu\right)\left(\mu+u_{2}-\mu p_{1}\right)}+\frac{c \omega \gamma S^{0}\left(\delta_{1} c_{1}+c_{2}\left(\mu+d+\sigma-\mu p_{1}\right)\right)}{\left(\mu+c_{1}+c_{2}\right)\left(\delta_{1}+\delta_{2}+\mu\right)\left(\mu+u_{2}-\mu p_{1}\right)} \frac{c \omega S^{0}}{\mu+d+u_{2}-\mu p_{1}}+\frac{\delta_{1} c \omega \gamma S^{0}}{\left(\mu+c_{1}+c_{2}\right)\left(\mu+u_{2}-\mu p_{1}\right)} \frac{c \omega \gamma S^{0}}{d \mu+c_{1}+c_{2}} & 0 \\ 0 & 0 \\ 0 & 0\end{array}\right]$

Then, effective reproduction number,

$$
R_{E f f}=\rho\left(F V^{-1}\right)=\frac{S^{0}\left(c \omega c_{1}\left(\mu+c_{1}+c_{2}\right)+\delta_{1} c \omega \gamma c_{1}+c \omega \gamma c_{2}\left(\mu+d+\sigma-\mu p_{1}\right)\right)}{\left(\mu+c_{1}+c_{2}\right)\left(\delta_{1}+\delta_{2}+\mu\right)\left(\mu+u_{2}-\mu p_{1}\right)}
$$

where $S^{0}=\left(\mu-\mu \theta+u_{2} / \tau+\mu+u_{2}-\mu \theta\right)$. After simplification, we get

$$
R_{E f f}=\frac{c \omega\left(\mu-\mu \theta+u_{2}\right)\left(c_{1}\left(\mu+c_{1}+c_{2}\right)+\delta_{1} \gamma c_{1}+\gamma c_{2}\left(\mu+d+\sigma-\mu p_{1}\right)\right)}{\left(\tau+\mu+u_{2}-\mu \theta\right)\left(\mu+c_{1}+c_{2}\right)\left(\delta_{1}+\delta_{2}+\mu\right)\left(\mu+u_{2}-\mu p_{1}\right)}
$$


Local stability analysis of the disease-free equilibrium point.

Theorem 3. The disease-free equilibrium point $E^{1}\left(S^{0}, E^{0}, A^{0}, C^{0}, T^{0}\right)$ given by

$$
E^{1}=\left(\frac{\mu-\mu \theta+u_{2}}{\tau+\mu+u_{2}-\mu \theta}, 0,0,0,0\right) .
$$

Disease-free equilibrium point of the dynamical system (1)-(5) is locally asymptotically stable, if $R_{E f f}<1$ and $E^{1}$ is unstable otherwise.

Proof.

$$
J\left(E^{1}\right)=\left[\begin{array}{ccccc}
-(\tau+\mu) & 0 & 0 & 0 & 0 \\
0 & -\left(c_{1}+c_{2}+\mu\right) & c \omega S^{0} & c \omega \gamma S^{0} & 0 \\
0 & c_{1} & -\left(\mu+\delta_{1}+\delta_{2}\right) & 0 & 0 \\
0 & c_{2} & \delta_{1} & -\left(\mu+d+\sigma-\mu p_{1}\right) & 0 \\
0 & 0 & c_{1} & u_{1} & -(\varphi+\mu)
\end{array}\right]
$$

Then, determinant of Jacobian matrix at $E^{1}$ is given by

$$
\Rightarrow\left|\begin{array}{ccccc}
-(\tau+\mu) & 0 & 0 & 0 & 0 \\
0 & -\left(c_{1}+c_{2}+\mu\right) & c \omega S^{0} & c \omega \gamma S^{0} & 0 \\
0 & c_{1} & -\left(\mu+\delta_{1}+\delta_{2}\right) & 0 & 0 \\
0 & c_{2} & \delta_{1} & \left(\mu+d+\sigma-\mu p_{1}\right) & 0 \\
0 & 0 & c_{1} & u_{1} & -(\varphi+\mu)
\end{array}\right|=0
$$

Let $\quad a=\tau+\mu, \mathrm{b}=c_{1}+c_{2}+\mu, \mathrm{c}=\mu+\delta_{1}+\delta_{2}, f=\mu+$ $d+\sigma-\mu p_{1}, e=\varphi_{1}+\mu$.

$$
\begin{aligned}
& \left|\begin{array}{ccccc}
-a-\lambda & 0 & 0 & 0 & 0 \\
0 & -b-\lambda & c \omega S^{0} & c \omega \gamma S^{0} & 0 \\
0 & c_{1} & -c-\lambda & 0 & 0 \\
0 & c_{2} & \delta_{1} & -f-\lambda & 0 \\
0 & 0 & c_{1} & u_{1} & -e-\lambda
\end{array}\right| \\
& \Rightarrow(-a-\lambda)(-e-\lambda)\left(-(f+\lambda)\left((b+\lambda)(c+\lambda)-c \omega c_{1} S^{0}\right)-c \omega \gamma S^{0}\left((c+\lambda) c_{2}+c_{1} \delta_{1}\right)\right)=0 \\
& \Rightarrow \lambda_{1}=-a, \lambda_{2}=-e,\left(-(f+\lambda)\left((b+\lambda)(c+\lambda)-c \omega c_{1} S^{0}\right)-c \omega \gamma S^{0}\left((c+\lambda) c_{2}+c_{1} \delta_{1}\right)\right)=0 \\
& \Rightarrow \lambda_{1}=-a, \lambda_{2}=-e, \lambda^{3}+(b+c+f) \lambda^{2}+\left(b c+f(b+c)+c \omega c_{1} S^{0}+c \omega \gamma c_{2} S^{0}\right) \lambda+c \omega c_{1} S^{0}+c^{2} \omega \gamma c_{2} S^{0}+c \omega \gamma c_{1} \delta_{1} S^{0}=0 .
\end{aligned}
$$

Then, letting $l_{1}=(b+c+f), l_{2}=(b c+f(b+$ $\left.c)+c \omega c_{1} S^{0}+c \omega \gamma c_{2} S^{0}\right), l_{3}=\omega c_{1} S^{0}+c^{2} \omega \gamma c_{2} S^{0}+c \omega \gamma c_{1} \delta_{1} S^{0}$,

$$
\lambda^{3}+(b+c+f) \lambda^{2}+\left(b c+f(b+c)+c \omega c_{1} S^{0}+c \omega \gamma c_{2} S^{0}\right) \lambda+c \omega c_{1} S^{0}+c^{2} \omega \gamma c_{2} S^{0}+c \omega \gamma c_{1} \delta_{1} S^{0}=\lambda^{3}+l_{1} \lambda^{2}+l_{2} \lambda+l_{3}
$$


We need to verify the following three conditions:
(a) $l_{1}, l_{2}, l_{3}>0$
(b) $l_{1} l_{2}-l_{3}>0$

Then, we have both $\lambda_{1}=-a, \lambda_{2}=-e$ which are negatives and the Routh-Hurwitz criterion and those inequalities in (a)-(b) imply that the characteristic equation at $E^{1}$ has only roots with negative real part, which certifies the local stability of $E^{1}$.

4.3. Endemic Equilibrium Point $\left(E_{1}\right)$. Endemic equilibrium point of the system of differential equation (1)-(5) is given by $E_{1}=\left(S^{*}, E^{*}, A^{*}, C^{*}, T^{*}\right)$ where

$$
\begin{aligned}
& S^{*} \frac{\left(\mu+c_{1}\right)\left(\mu+c_{2}\right)\left(\mu+\delta_{2}-p_{1} \mu+u_{2}\right)}{c \omega c_{1}\left(\mu+\delta_{2}+\delta_{1}-p_{1} \mu+u_{2}\right)} E^{*}=\frac{c \omega\left(A^{*}+\gamma C^{*}\right) S^{*}}{\mu+c_{1}+c_{2}}, A^{*}=\frac{c \omega c_{1} \gamma C^{*} S^{*}}{1-c \omega c_{1} S^{*}}, \\
& C^{*}=c_{2} c \omega\left(\frac{\left(A^{*}+\gamma C^{*}\right) S^{*}}{\left(\mu+c_{1}+c_{2}\right)\left(\mu+d+\sigma-\mu p_{1}\right)}\right)+\frac{c_{1} \delta_{1} c \omega \theta C^{*} S^{*}}{\left(1-c \omega c_{1} S^{*}\right)\left(\mu+\sigma-\mu p_{1}\right)}, \\
& T^{*}=\frac{\tau}{(\varphi+\mu)}\left(\frac{c_{2}\left(c \omega\left(A^{*}+\gamma C^{*}\right) S^{*}\right)}{\left(c_{1}+c_{2}+\mu\right)\left(\mu+\sigma-\mu p_{1}\right)}+\frac{c_{1} \delta_{1} c \omega \gamma C^{*} S^{*}}{\left(1-c \omega c_{1} S^{*}\right)\left(\mu+\sigma-\mu p_{1}\right)}\right) .
\end{aligned}
$$

4.4. Local Stability of Endemic Equilibrium Point $\left(E_{1}\right)$. Endemic equilibrium points are steady-state solutions where the disease persists in the population (all state variables are positive). We use the Jacobean matrix and Routh-Hurwitz criterion to prove the existence of at least one locally stable endemic equilibrium point for $R_{E f f}>1$. When $R_{E f f}>1$, it is expected that the disease would be able to invade in the population.

Theorem 4. The endemic equilibrium point $E_{1}=(S *, E *, A *, C *, T *)$ of the HBV model (1)-(5) is locally asymptotically stable (LAS) if $R_{\text {eff }}>1$.

$$
J\left(E_{1}\right)=\left[\begin{array}{ccccc}
a & b & b & c & b \\
0 & e & 0 & 0 & 0 \\
0 & c_{1} & f & 0 & 0 \\
0 & c_{2} & \delta_{1} & -g & 0 \\
0 & 0 & 0 & \sigma & -h
\end{array}\right],
$$

where $a=c \omega\left(A^{*}+\gamma C^{*}\right)+\tau+\mu+\mu \theta-u_{2}, b=\mu \theta-u_{2}, c=$ $\mu p_{2}-u_{2}-\mu p_{1}, e=c_{1}+c_{2}+\mu, \mathrm{f}=\mu+\delta_{1}+\delta_{2}, g=\mu+\sigma-\mu$ $p_{1}, h=\varphi_{1}+\mu$.

Then, determinant of Jacobian matrix is given by

$$
\left|\begin{array}{ccccc}
a-\lambda & b & b & c & b \\
0 & e-\lambda & 0 & 0 & 0 \\
0 & c_{1} & f-\lambda & 0 & 0 \\
0 & c_{2} & \delta_{1} & -g-\lambda & 0 \\
0 & 0 & 0 & \sigma & -h-\lambda
\end{array}\right|=0 .
$$

Then, the characteristic equation at $E_{1}$ is $(\lambda+a b g)\left(\lambda^{4}+\right.$ $\left.k_{1} \lambda^{3}+k_{2} \lambda^{2}+k_{3} \lambda+k_{4}\right)=0$ where $\lambda_{1}=-a b g$,

$$
\begin{aligned}
k_{1} & =a+e+f+g+h, k_{2}=a c+e f-a g-b h-b g, k_{3}=c f h+a c g+c f h-b c e-b f g, k_{4} \\
& =a e f h+a b g h+b c f g-a f g h-c e f h .
\end{aligned}
$$

We need to verify the following three conditions:

(c) $k_{1}, k_{2}, k_{3}, k_{4}>0$

(d) $k_{1} k_{2}-k_{3}>0$

(e) $k_{3}\left(k_{1} k_{2}-k_{3}\right)-k_{1}^{2} k_{4}>0$

It is easy to see those conditions (a) and (b) are satisfied. After computations, we can prove that $k_{3}\left(k_{1} k_{2}-k_{3}\right)-k_{1}^{2} k_{4}>0$ is also valid. The Routh-Hurwitz criterion and those inequalities in (a)-(c) imply that the characteristic equation at $E_{1}$ has only roots with negative real part, which certifies the local stability of $E_{1}$.

Globally Asymptotically Stability (GAS) of the DiseaseFree Equilibrium Point $\left(E^{1}\right)$.

Theorem 5. The disease-free equilibrium $E^{1}=\left(\mu-\mu \theta+u_{2} / \tau+\mu+u_{2}-\mu \theta, 0,0,0,0\right)$ is globally asymptotically stable in the feasible region $\Omega$ if $R_{E f f}<1$. 
Proof. To proof this theorem, we first developed a Lyapunov function, technically.

$$
L=\left(\frac{c w+\gamma \delta_{1}}{k_{1} k_{2} k_{3}}\right) E+\sigma A+k_{2} C,
$$

where $k_{1}=c_{1}+c_{2}+\mu, k_{2}=\delta_{1}+\delta_{2}+\mu, k_{3}=\sigma+\mu-\mu p_{1}$.
Then, differentiating $L$ both sides leads to

$$
\frac{\mathrm{d} L}{\mathrm{~d} t}=\left(\frac{c w+\gamma \delta_{1}}{k_{1} k_{2} k_{3}}\right) \lambda S+\sigma \frac{\mathrm{d} A}{\mathrm{~d} t}+k_{2} \frac{\mathrm{d} C}{\mathrm{~d} t} .
$$

Substituting expression for $\mathrm{d} E / \mathrm{d} t, \mathrm{~d} A / \mathrm{d} t$, and $\mathrm{d} C / \mathrm{d} t$ from equation (36),

$$
\frac{d L}{d t}=\left(\frac{c w+\gamma \delta_{1}}{k_{1} k_{2} k_{3}}\right) \lambda S-\left(c_{1}+c_{2}+\mu\right) E+\left(c_{1} E-\left(\delta_{1}+\delta_{2}+\mu\right) A\right)+c_{2} E+\delta_{1} A-\left(\sigma+\mu-\mu p_{1}\right) C .
$$

By collecting like terms of (37), we get

$$
\begin{aligned}
& \frac{\mathrm{d} L}{\mathrm{~d} t}=\left(\frac{c w+\gamma \delta_{1}}{k_{1} k_{2} k_{3}}\right) \lambda S+\left(\delta c_{1}-\mu\right) E+\left(\delta_{2}+\mu\right) A-\left(\sigma+\mu-\mu p_{1}\right) C, \\
& \frac{\mathrm{d} L}{\mathrm{~d} t}=\left(R_{E f f}\left(\delta c_{1}-\mu\right)\right) \lambda S\left(\delta c_{1}-\mu\right) E+\left(\delta_{2}+\mu\right) A-\left(\sigma+\mu-\mu p_{1}\right) C, \\
& \frac{d L}{d t} \leq\left(R_{E f f}-1\right)\left(\delta_{2}+\mu\right) A .
\end{aligned}
$$

So, $\mathrm{d} L / \mathrm{d} t \leq 0$ if $R_{E f f} \leq 1$. Furthermore, $\mathrm{d} L / \mathrm{d} t=0$ which implies $A=0$ which leads to $E=C=0$ or $R_{E f f}=1$.

Hence, $L$ is Lyapunov function on $\Omega$ and the largest compact invariant set in $\{(S, E, A, C, T) \in \Omega, \mathrm{d} L / \mathrm{d} t=0\}$ is the singleton $\left(S^{0}, 0,0,0,0\right)$ where $S^{0}=\left(\mu-\mu \theta+u_{2} / \tau+\mu+u_{2}-\mu \theta\right)$. Therefore, by LaSalle's invariance principal [30], every solution to equations of model (1)-(5) with initial conditions in $\Omega$ which approaches the disease-free equilibrium at $t$ (time) tends to infinity $(t \longrightarrow \infty)$ whenever $R_{E f f} \leq 1$. Hence, the disease-free equilibrium is globally asymptotically stable.

\subsection{Global Stability of Endemic Equilibrium Point (EEP), $E_{1}$}

Theorem 6. The endemic equilibrium point $E_{1}=\left(S^{*}, E^{*}, A^{*}, C^{*}, T^{*}\right)$ is globally asymptotically stable in the feasible region $\Omega$ if $R_{E f f}>1$.

Let the endemic equilibrium of our normalized model system (1)-(5) be denoted by $E_{1}$. It is obtained by $\left(S^{*}, E^{*}, A^{*}, C^{*}, T^{*}\right)$ setting the right-hand side of each equation of the normalized model system (1)-(5) equal to zero and solving for the state variables in terms of the force of $\lambda=c \omega(\gamma C+A)$. That is, $E_{1}=\left(S^{*}, E^{*}, A^{*}, C^{*}, T^{*}\right)$ where

$$
\begin{aligned}
S^{*} & =\frac{\left(\mu+\lambda_{1}\right)\left(\mu+\lambda_{2}\right)\left(\mu+\lambda_{3}-p_{1} \mu+u_{2}\right)}{c \omega \lambda_{1}\left(\mu+\lambda_{3}+\gamma \lambda_{2} p_{3}-p_{1} \mu+u_{2}\right)} E^{*}=\frac{c \omega\left(A^{*}+\gamma C^{*}\right) S^{*}}{\mu+c_{1}+c_{2}}, A^{*}=\frac{c \omega c_{1} \gamma C^{*} S^{*}}{1-c \omega c_{1} S^{*}} \\
C^{*} & =c_{2} c \omega\left(\frac{\left(A^{*}+\gamma C^{*}\right) S^{*}}{\left(\mu+c_{1}+c_{2}\right)\left(\mu+d+u_{1}-\mu p_{1}\right)}\right)+\frac{c_{1} \delta_{1} c \omega \theta C^{*} S^{*}}{\left(1-c \omega c_{1} S^{*}\right)\left(\mu+\sigma-\mu p_{1}\right)} \\
T^{*} & =\frac{u_{1}}{(\varphi+\mu)}\left(\frac{c_{2}\left(c \omega\left(A^{*}+\gamma C^{*}\right) S^{*}\right)}{\left(c_{1}+c_{2}+\mu\right)\left(\mu+d+\sigma-\mu p_{1}\right)}+\frac{c_{1} \delta_{1} c \omega \gamma C^{*} S^{*}}{\left(1-c \omega c_{1} S^{*}\right)\left(\mu+d+\sigma-\mu p_{1}\right)}\right)
\end{aligned}
$$

Now, we can show the existence of endemic equilibrium points using the force of infection $\lambda=c \omega(\gamma C+A)$.

Then, $\lambda=c \omega(\gamma C+A)$ this implies. $\lambda=c \omega\left(\gamma C^{*}+A^{*}\right) \quad$ substituting $A^{*}=\left(c \omega c_{1} \gamma C^{*} S^{*}\right) / 1-$ $c \omega c_{1} S^{*}, C^{*}=c_{2} c \omega\left(\left(A^{*}+\gamma C^{*}\right) S^{*} /\left(\mu+c_{1}+c_{2}\right)\left(\mu+d+u_{1}-\right.\right.$ $\left.\left.\mu p_{1}\right)\right)+c_{1} \delta_{1} c \omega \theta C^{*} S^{*} /\left(1-c \omega c_{1} S^{*}\right)\left(\mu+\sigma-\mu p_{1}\right)$, we get 


$$
\begin{aligned}
& \lambda=c \omega\left(\gamma\left(c_{2} c \omega\left(\frac{\left(A^{*}+\gamma C^{*}\right) S^{*}}{\left(\mu+c_{1}+c_{2}\right)\left(\mu+d+u_{1}-\mu p_{1}\right)}\right)+\frac{c_{1} \delta_{1} c \omega \theta C^{*} S^{*}}{\left(1-c \omega c_{1} S^{*}\right)\left(\mu+\sigma-\mu p_{1}\right)}\right)+\frac{c \omega c_{1} \gamma C^{*} S^{*}}{1-c \omega c_{1} S^{*}}\right) \\
& \Rightarrow \lambda=c \omega C^{*} S^{*}\left(\left(\frac{c_{2} \lambda}{\left(\mu+c_{1}+c_{2}\right)\left(\mu+d+u_{1}-\mu p_{1}\right)}\right)+\frac{c_{1} \delta_{1}}{\left(1-c \omega c_{1} S^{*}\right)\left(\mu+\sigma-\mu p_{1}\right)}+\frac{c_{1} \gamma}{1-c \omega c_{1} S^{*}}\right), \\
& \lambda\left(1-\frac{c_{2} c \omega C^{*} S^{*}}{\left(\mu+c_{1}+c_{2}\right)\left(\mu+d+u_{1}-\mu p_{1}\right)}\right)=c_{1} c \omega C^{*} S^{*}\left(\frac{\delta_{1}}{\left(1-c \omega c_{1} S^{*}\right)\left(\mu+\sigma-\mu p_{1}\right)}+\frac{\gamma}{1-c \omega c_{1} S^{*}}\right) \\
& \Rightarrow \lambda\left(1-R_{E f f}\right)=c_{1} c \omega C^{*} S^{*}\left(\frac{\gamma}{\left(1-c \omega c_{1} S^{*}\right)\left(\mu+\sigma-\mu p_{1}\right)}+\frac{\gamma}{1-c \omega c_{1} S^{*}}\right) .
\end{aligned}
$$

Letting $\quad D_{1}=c_{1} c \omega C^{*} S^{*}\left(\left(\delta_{1} /\left(1-c \omega c_{1} S^{*}\right)(\mu+\sigma-\mu\right.\right.$ $\left.\left.\left.p_{1}\right)\right)+\gamma / 1-c \omega c_{1} S^{*}\right)$ which is less than zero (negative).

Then, for $\lambda\left(1-R_{E f f}\right)=D_{1}$ where $D_{1}$ is clearly less than zero (negative), to have positive eigenvalue, $1-R_{E f f}$ should be negative meaning that $R_{E f f}>1$. This shows existence of unique endemic equilibrium point which is globally asymptotically stable, as shown in Figure 2 graphically. This ends the proof.

\section{Sensitivity Analysis}

To determine the most optimum approach in suppressing the number of infected individuals, one needs to identify several factors that contribute to the transmission of the virus and its prevalence. In this section, the study calculates the sensitivity index of each parameter model that correlates with the effective reproductive number, $R_{E f f}$. This index provides information about the importance of each parameter to the model representing the transmission of HBV. The index is used to identify the parameter that has the most significant impact on $R_{\mathrm{Eff}}$ which is later served as the intervention target. The parameter with a high impact in $R_{\text {Eff }}$ shows that the parameter has a dominant influence on the endemicity of HBV. We carried out the sensitivity analysis to determine the model robustness to parameter values. The normalized forward sensitivity index of a variable $R_{\text {Eff }}$ that depends differentiable on a parameter $p$ is defined as $\operatorname{SI}(P)=\left(\partial R_{e f f} / \partial p\right) \times\left(p / R_{e f f}\right)$ [31]. The parameter with higher sensitivity index magnitude is/are more influential than that with smaller magnitude of sensitive index. The sign of the sensitivity indices of $R_{\mathrm{Eff}}$ with respect to the parameters shows the positive or negative impact of the parameter on $R_{\mathrm{Eff}}$. That is, if the sign of the sensitivity indices is positive, then the value of $R_{\mathrm{Eff}}$ increases whenever the value of the parameter increases and if the sign of the sensitivity indices is negative, then the value of $R_{\text {Eff }}$ decreases whenever the value of the parameter increases.

The resulting sensitivity indices of $R_{\mathrm{Eff}}$ to the sixteen different parameters in the model are shown in Table 1 in the order from the most sensitive to least. From the sensitivity index of the model, we consider that the most sensitive parameter is $c_{2}$, which is transfer rate of exposed class to acute infective class. The least sensitive parameter is $u_{2}$, which is rate of loss of immunity.
The parameters $c_{2}$ and $c_{1}$ have a positive sensitivity index, while the parameters $\theta$ and ? have a negative sensitivity index. Parameters with a positive sensitivity index represent the positive significance in the increase of effective reprodzuction numbers. Thereby, increasing (or decreasing) the value of the parameter, while the other parameters' value remaining the same will contribute to the increases (or decreases) in the effective reproduction numbers. Parameters with a negative sensitivity index represent the negative significance to the increase of effective reproduction numbers. In other words, increasing (or decreasing) the value of the parameter, while the other parameters' value remains the same, will contribute to the decreases (or increases) in the effective reproduction numbers. Sensitivity index shows that the transfer rate from exposed class to acute infective class and transfer rate from exposed class to chronic infective are the most dominant parameters contributing to the transmission of $\mathrm{HBV}$. On the one hand, the vaccination rate and treatment rate are the parameters that suppress the transmission of the disease the most.

Since $\operatorname{SI}\left(c_{2}\right)=\left(\partial R_{\mathrm{Eff}} / \partial c_{2}\right) \times\left(c_{2} / R_{\mathrm{Eff}}\right)=+1.4761$, increasing (or decreasing) the parameter $c_{2}$ by $10 \%$ will result in increases (or decreases) of the value effective reproduction number by $14.761 \%$. The result that $\operatorname{SI}(\theta)=\left(\partial R_{\mathrm{Eff}} / \partial \theta\right) \times$ $\left(\theta / R_{\mathrm{Eff}}\right)=-1.3287$ signifies that increasing (or decreasing) the parameter $\theta$ by $10 \%$ will lead to a decrease (or increases) in the value of effective reproduction number by $13.287 \%$. Interrupting transfer rate from exposed class to both acute infective and chronic infective and maximizing vaccination for the population and treatment for chronically infected population are crucial.

\section{Numerical Simulation and Discussion}

Here, we discuss effect of each parameter to the value of effective reproduction number to determine their contribution for transmission of HBV.

Figure 3 shows the HBV effective reproduction number simulation at variable transfer rate from exposed class to chronic infective class $c_{1}$, and from the graph, we see that $\mathrm{HBV}$ infection dies out at HBV vaccination rate $c_{1}<0.267$. This shows that by keeping transfer rate from exposed class to chronic infective class less than 0.267 , it is possible to decrease transmission of $\mathrm{HBV}$. 


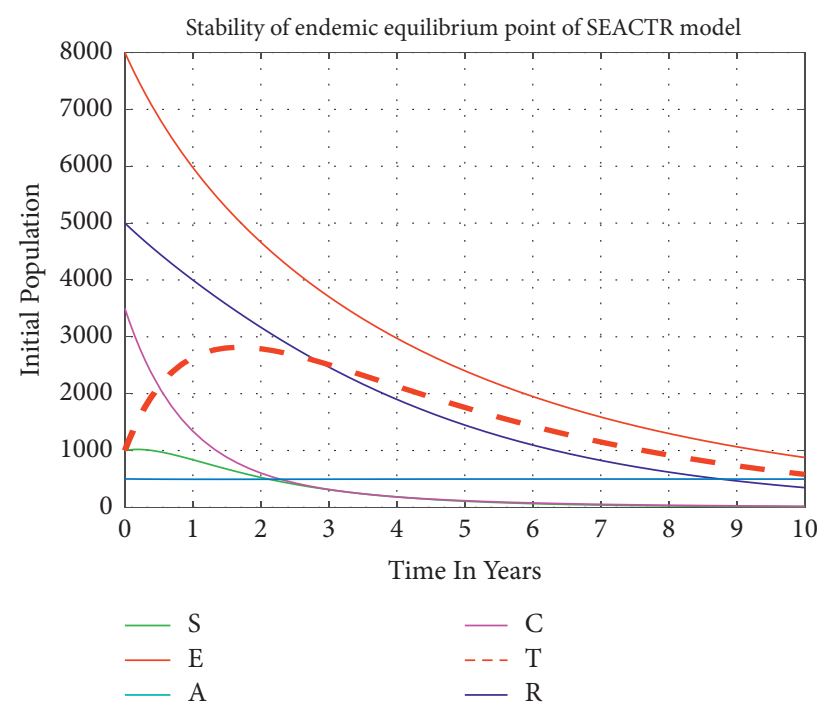

FIgURE 2: Behavior of SEACTR model at endemic equilibrium point.

TABLE 1: Sensitivity indices of the model parameters.

\begin{tabular}{lc}
\hline Sensitivity index & Value \\
\hline $\operatorname{SI}\left(c_{2}\right)=\left(\partial R_{\mathrm{Eff}} / \partial c_{2}\right) \times\left(c_{2} / R_{\mathrm{Eff}}\right)$ & +1.4761 \\
$\operatorname{SI}\left(c_{1}\right)=\left(\partial R_{\mathrm{Eff}} / \partial c_{1}\right) \times\left(c_{1} / R_{\mathrm{Eff}}\right)$ & +1.3769 \\
$\mathrm{SI}(\theta)=\left(\partial R_{\mathrm{Eff}} / \partial \theta\right) \times\left(\theta / R_{\mathrm{Eff}}\right)$ & -1.3287 \\
$\mathrm{SI}(\sigma)=\left(\partial R_{\mathrm{Eff}} / \partial \sigma\right) \times\left(\sigma / R_{e f f}\right)$ & -1.0025 \\
$\operatorname{SI}(c)=\left(\partial R_{\mathrm{Eff}} / \partial c\right) \times\left(c / R_{\mathrm{Eff}}\right)$ & +1 \\
$\operatorname{SI}(\omega)=\left(\partial R_{\mathrm{Eff}} / \partial \omega\right) \times\left(\omega / R_{\mathrm{Eff}}\right)$ & +1 \\
$\operatorname{SI}(\varphi)=\left(\partial R_{\mathrm{Eff}} / \partial \varphi\right) \times\left(\varphi / R_{\mathrm{Eff}}\right)$ & +0.9138 \\
$\operatorname{SI}\left(\delta_{1}\right)=\left(\partial R_{\mathrm{Eff}} / \partial \delta_{1}\right) \times\left(\delta_{1} / R_{\mathrm{Eff}}\right)$ & -0.7338 \\
$\operatorname{SI}\left(\delta_{2}\right)=\left(\partial R_{\mathrm{Eff}} / \partial \delta_{2}\right) \times\left(\delta_{2} / R_{\mathrm{Eff}}\right)$ & -0.5968 \\
$\operatorname{SI}\left(\varphi_{1}\right)=\left(\partial R_{\mathrm{Eff}} / \partial \varphi_{1}\right) \times\left(\varphi_{1} / R_{\mathrm{Eff}}\right)$ & -0.4286 \\
$\operatorname{SI}(d)=\left(\partial R_{\mathrm{Eff}} / \partial d\right) \times\left(d / R_{\mathrm{Eff}}\right)$ & +0.3748 \\
$\operatorname{SI}(\mu)=\left(\partial R_{\mathrm{Eff}} / \partial \mu\right) \times\left(\mu / R_{e f f}\right)$ & +0.3647 \\
$\operatorname{SI}(\tau)=\left(\partial R_{\mathrm{Eff}} / \partial \tau\right) \times\left(\tau / R_{\mathrm{Eff}}\right)$ & +0.3593 \\
$\operatorname{SI}\left(\varphi_{1}\right)=\left(\partial R_{\mathrm{Eff}} / \partial \varphi_{1}\right) \times\left(\varphi_{1} / R_{\mathrm{Eff}}\right)$ & +0.33962 \\
$\operatorname{SI}\left(p_{1}\right)=\left(\partial R_{\mathrm{Eff}} / \partial p_{1}\right) \times\left(p_{1} / R_{\mathrm{Eff}}\right)$ & +0.33593 \\
$\operatorname{SI}\left(u_{2}\right)=\left(\partial R_{\mathrm{Eff}} / \partial u_{2}\right) \times\left(u_{2} / R_{\mathrm{Eff}}\right)$ & +0.3297 \\
\hline
\end{tabular}

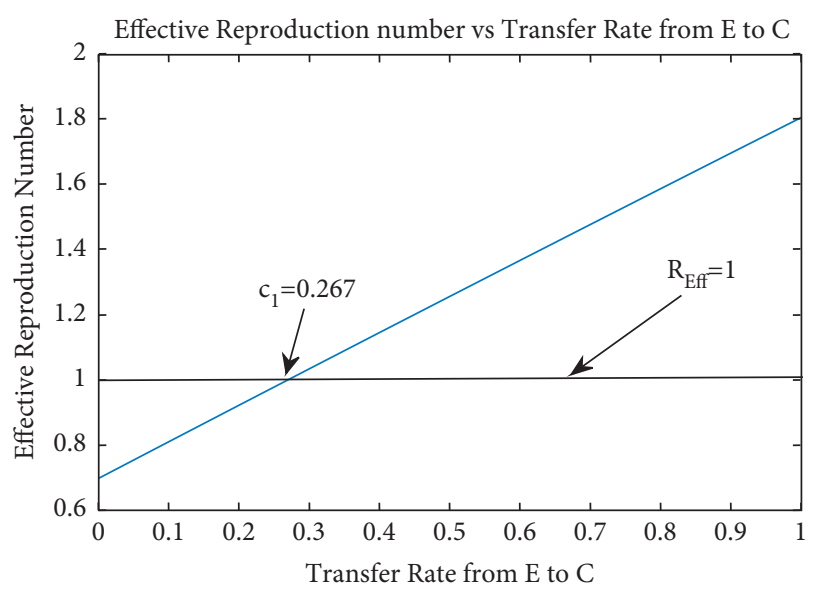

FIGURE 3: Behavior of effective reproduction number in relation to $c_{1}$.
Figure 4 shows the HBV effective reproduction number simulation at variable contact rate $c$, and from the graph, we see that $\mathrm{HBV}$ infection dies out at $\mathrm{HBV}$ contact rate $c<0.32$.This shows that by keeping contact rate less than 0.32 , it is possible to decrease transmission of $\mathrm{HBV}$.

Figure 5 shows the HBV effective reproduction number simulation at variable vaccination rate $\theta$, and from the graph, we see that $\mathrm{HBV}$ infection dies out at $\mathrm{HBV}$ vaccination rate $\theta>0.41$. This shows that by increasing number of vaccinated populations and keeping it greater than 0.41 , it is possible to decrease transmission of HBV.

Figure 6 shows the HBV effective reproduction number simulation at variable treatment rate $\sigma$, and from the graph, we see that HBV infection dies out at HBV treatment rate $\sigma>0.682$. This shows that by increasing number of treated chronic infective populations and keeping it greater than 0.682 , it is possible to decrease transmission of $\mathrm{HBV}$.

Figure 7 shows the HBV effective reproduction number simulation at variable transfer rate from exposed class to acute infective class $c_{2}$, and from the graph, we see that HBV infection dies out at $\mathrm{HBV}$ transfer rate from exposed class to acute infective class $c_{2}<0.192$. This shows that by keeping transfer rate from exposed class to acute infective class less than 0.192, it is possible to decrease transmission of HBV.

In Figure 8 , we considered $R_{0}$-basic reproduction number, $R_{V O n l y}$ - reproduction number with intervention of vaccination only, $R_{\text {TOnly }}$ - reproduction number with intervention of treatment only, and $R_{V T}-$ reproduction number with intervention of vaccination and treatment (effective reproduction number). It is shown that $R_{0}>R_{\text {TOnly }}>R_{V O n l y}>R_{V T}$. This means reproduction number of HBV disease without intervention is greater than reproduction number with intervention of treatment only, reproduction number with intervention of treatment only is greater than reproduction number with intervention of vaccination only, and reproduction number with intervention of vaccination only is greater than reproduction number with intervention of treatment and vaccination 


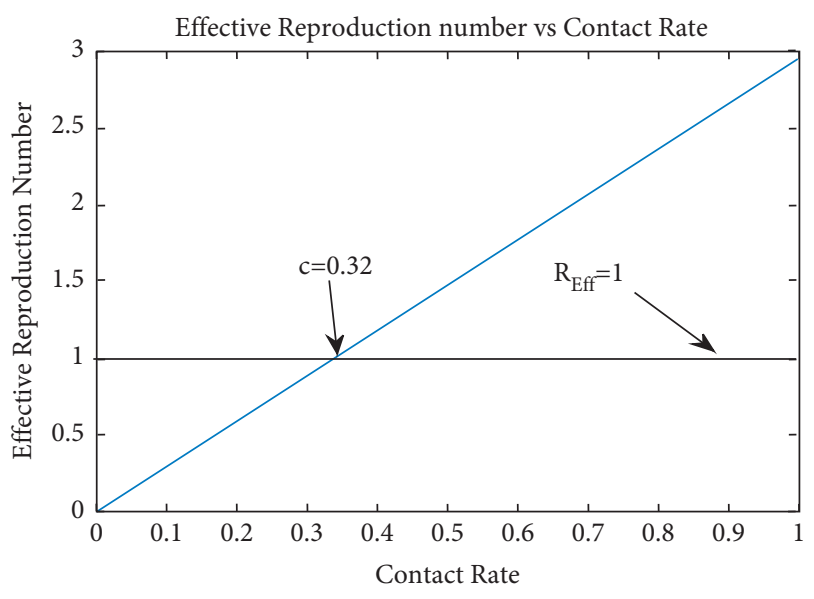

FIGURE 4: Behavior of effective reproduction number in relation to contact rate, $c$.

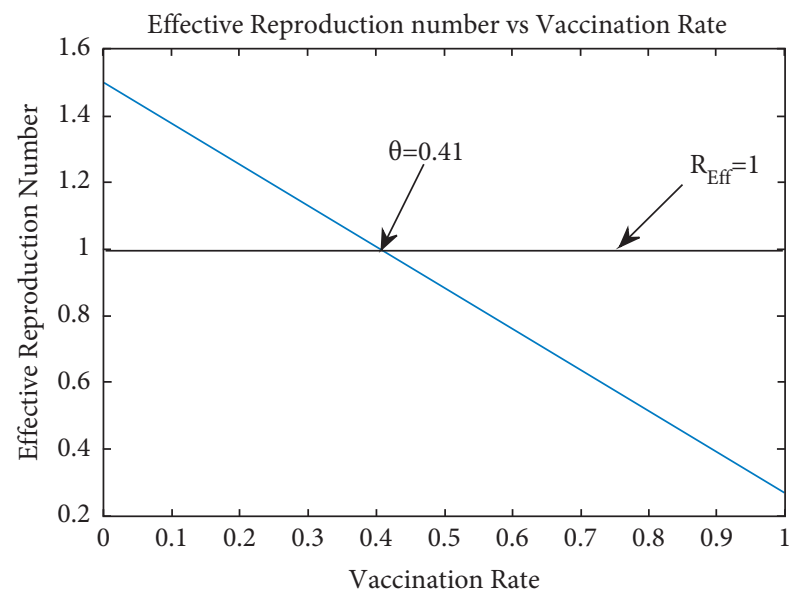

FIgURE 5: Behavior of effective reproduction number in relation to vaccination rate, $\theta$.

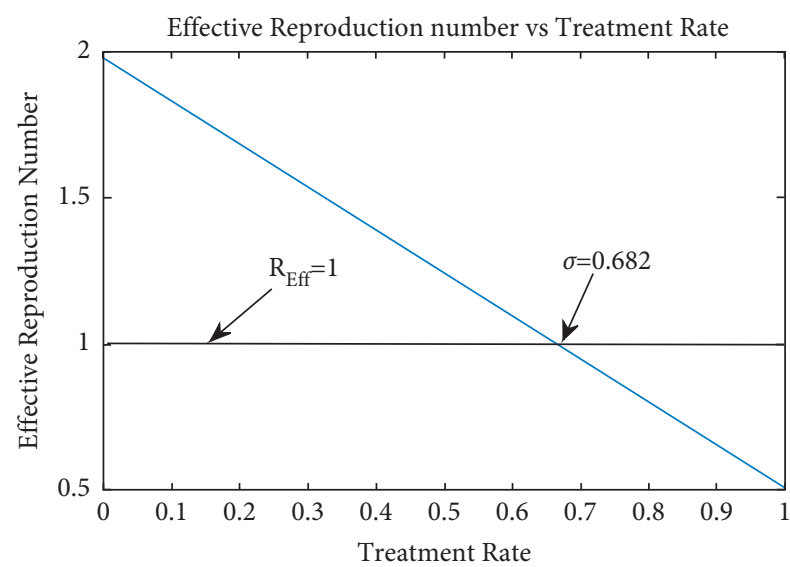

FIGURE 6: Behavior of effective reproduction number in relation to treatment rate, $\sigma$

(effective reproduction number). This confirms that using intervention of both vaccination and treatment plays best role in decreasing reproduction number of HBV disease.

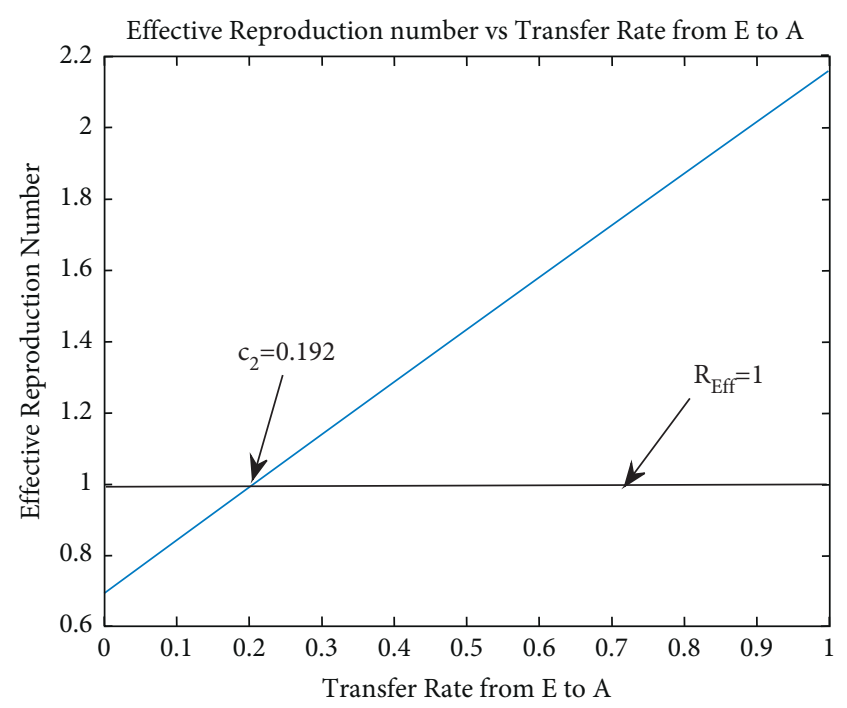

FIGURE 7: Behavior of effective reproduction number in relation to $c_{2}$.

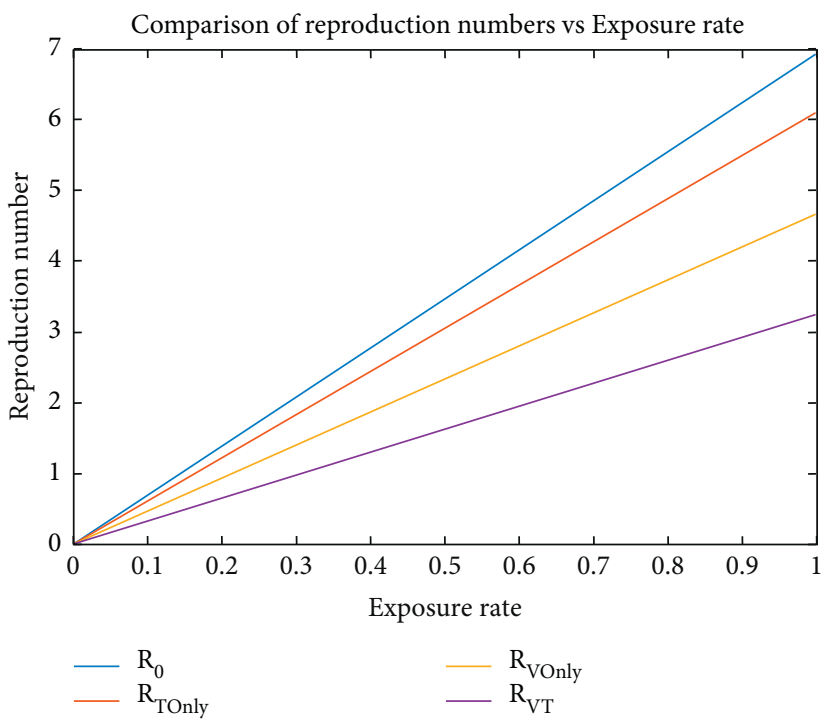

FIGURE 8: Basic reproduction number and reproduction number with interventions vs exposure rate.

As it is shown in Figure 9, when effective reproduction number is less than unity, number of susceptible and recovered population increases, whereas number of infective classes decreases. This confirms that keeping effective reproduction number less than unity helps to decrease the transmission of HBV implying decrease of infective population and increase of noninfective population in the society.

Figure 2 is plotted using the values $R_{\mathrm{Eff}}=3.513$ at $c_{1}=$ $0.67, c_{2}=0.281$ with all other parameters as given in Table 2 . The simulation in Figure 2 shows the stability of the HBV model endemic equilibrium point. From Figure 2, in the long run, the convergence of the solutions is observed at the values greater than 10 years. 


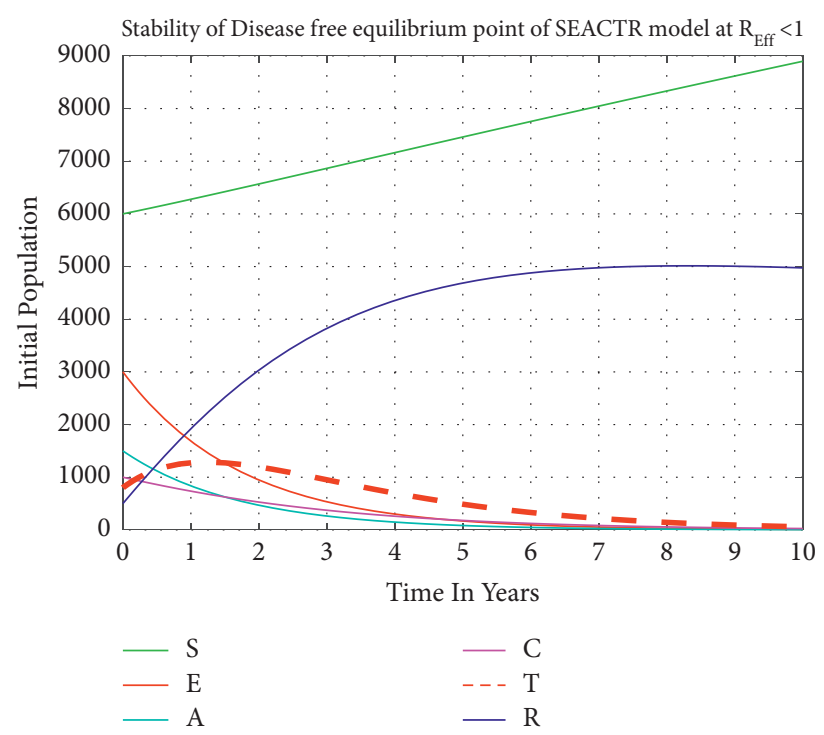

FIGURE 9: Behavior of SEACTR model when $R_{\mathrm{Eff}}<1$.

TABLE 2: Parameters and their values used in numerical simulation.

\begin{tabular}{lccc}
\hline Parameter & Description & Values Range & Reference \\
\hline$c$ & Contact rate & 0.33 & {$[23]$} \\
$\omega$ & Probability of acquiring HBV infectious per contact with one infectious individual & 0.32 & {$[24]$} \\
$\gamma$ & Level of infectiousness of chronically infected population & 0.5 & {$[23]$} \\
$\theta$ & Vaccination rate & 0.52 & 0.27 \\
$\mu$ & Birth (Death) rate & 0.11 & {$[25]$} \\
$p_{1}$ & Probability of infected newborns & 0.082 \\
$c_{1}$ & Transfer rate from $E$ to C & {$[24]$} \\
$c_{2}$ & Transfer rate from $E$ to A & {$[24]$} \\
$\sigma$ & Treatment rate & {$[25]$} \\
$\sigma \delta_{1}$ & Rate of moving from acute to chronic & 0.13 & {$[27]$} \\
$\delta_{2}$ & Rate of moving from acute to recovery & 0.475 \\
$\varphi$ & Recovery rate & 0.4 & {$[24]$} \\
$d$ & HBV disease-induced death rate & {$[23]$} & 0.32 \\
$\tau$ & Proportions of susceptible move to recovered class by vaccination & 0.175 & Assumed \\
\hline
\end{tabular}

Figure 10 is plotted using MATLAB ode45 program under consideration of the effective reproduction numbers being less than a unity and shows the behavior of the infectious classes of the HBV model (1)-(6) at $R_{\text {Eff }}<1$. The simulation given in Figure 10 shows that each of infectious classes $(E, A, C)$ is converging to the disease-free equilibrium point of the model. This is obtained when $R_{\mathrm{Eff}}=0.765$ at $c_{1}=0.00437, c_{2}=0.000725$ with all other parameters as given in Table 2 . This indicates that the disease-free equilibrium point of the HBV model is globally asymptotically stable. From Figure 10, it is understood that making effective reproduction number less than a unity leads to convergence of infective population to zero. This confirms that if $R_{\mathrm{Eff}}<1$, then the HBV disease dies out in ten years.

As it is observed from Figure 11, as we decreased transfer rate from exposed class to acutely infected class from 0.5 to 0.14 , the number of acute infective population also decreases from 1,250 to 730 . This shows that by decreasing transfer rate, it is possible to decrease acutely infected population.
This can be successful by educating the society to have best understanding about means of transmission of HBV disease.

As it is shown in Figure 12, as we decreased transfer rate from exposed class to chronic infective class from 0.59 to 0.12 , the number of chronically infected population also decreases from 950 to 700 . This shows that as transfer rate increases, the number of chronically infected population also increases. Then, to decrease the number of chronically infected population, transfer rate should be decreased by educational campaign in the society.

From Figure 13, we can understand that as treatment rate increases from 0.34 to 0.59 , the number of chronically infected population decreases faster. So, in order to decrease chronically infected population, we should give early treatment for individuals who are chronically infected by HBV disease.

From Figure 14, it is observed that as we increased vaccination rate from 0.13 to 0.79 , then the number of chronic infective population decreases faster and converges 


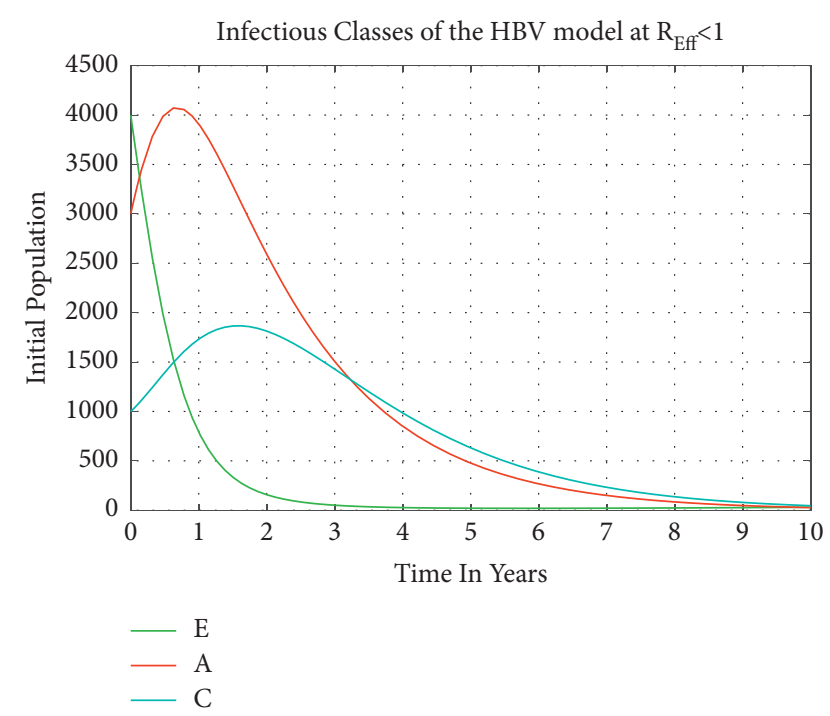

Figure 10: Behavior of infectious classes of SEACTR model when $R_{\text {Eff }}<1$.

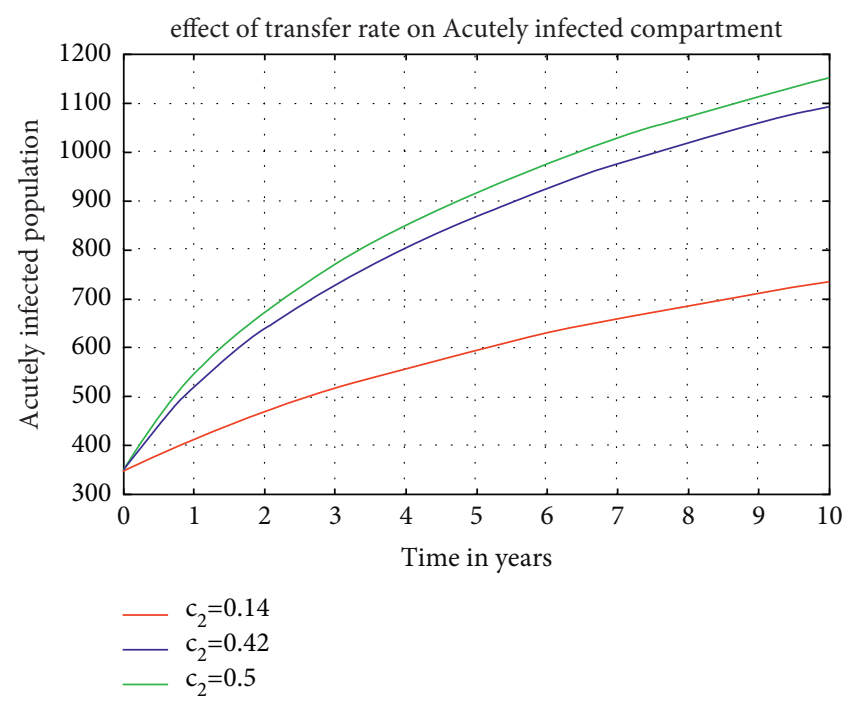

FIGURE 11: Effect of transfer rate on acutely infected population.

to zero. Then, increasing vaccination rate enables to decrease chronically infected individuals. So, vaccinating new born population plays a vital role in decreasing chronically infected individuals in the society.

As it is shown in Figure 15, as the vaccination rate is increased from 0.14 to 0.59 , the number of acutely infected population falls fast. This shows that vaccinating the population has a great importance in decreasing acutely infected individuals in the society. So, increasing vaccination rate of the population enables to decrease number of acutely infected individuals.

From Figure 16, it is understood that as the rate of vaccination increased from 0.12 to 0.549 , the number of susceptible populations also increases from 1, 350 to 1,650 . This shows that vaccination decreases the number of

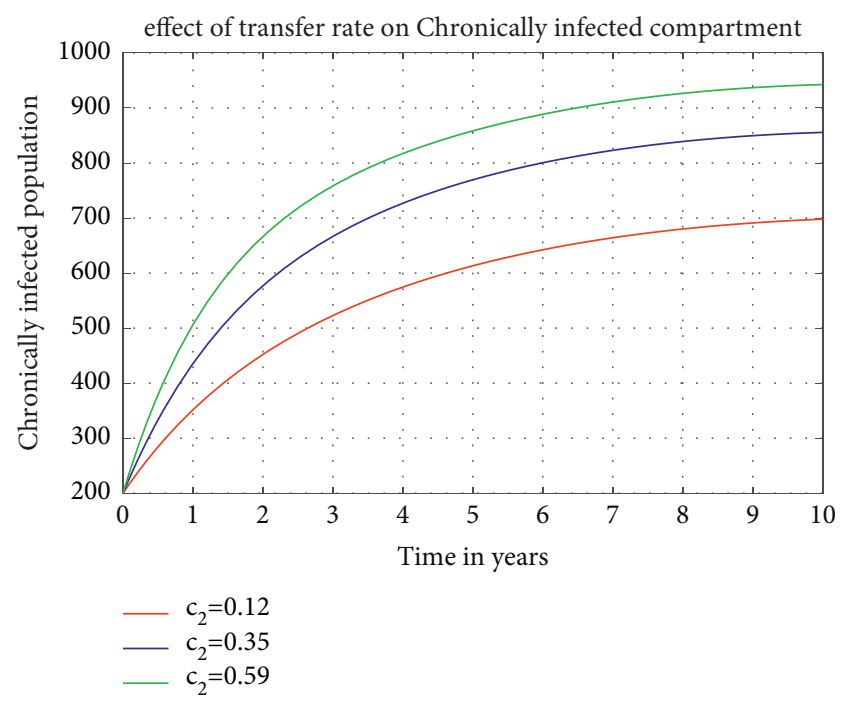

FIgURE 12: Effect of transfer rate on chronically infected compartment.

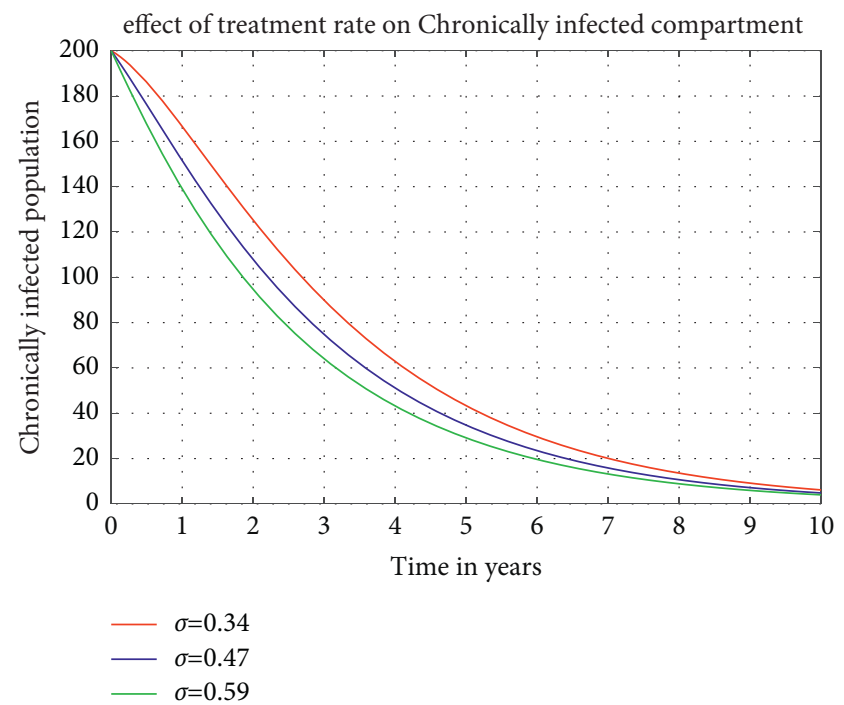

FIgURE 13: Effect of treatment rate on chronically infected compartment.

infected populations and leads to increment of susceptible population confirming that vaccination is one of the best control strategies for HBV.

As it is understood from Figure 17, as we increase the rate of treatment from 0.012 to 0.49 , the number of susceptible populations grows faster. This shows treatment for infected population also plays a vital role in decreasing infected population and in reverse in increasing the number of susceptible populations.

As it is shown in Figure 18, as we increase vaccination rate from 0.112 to 0.7549 , the number of exposed populations decreases faster. This shows that vaccination intervention plays crucial role in decreasing exposed population. 


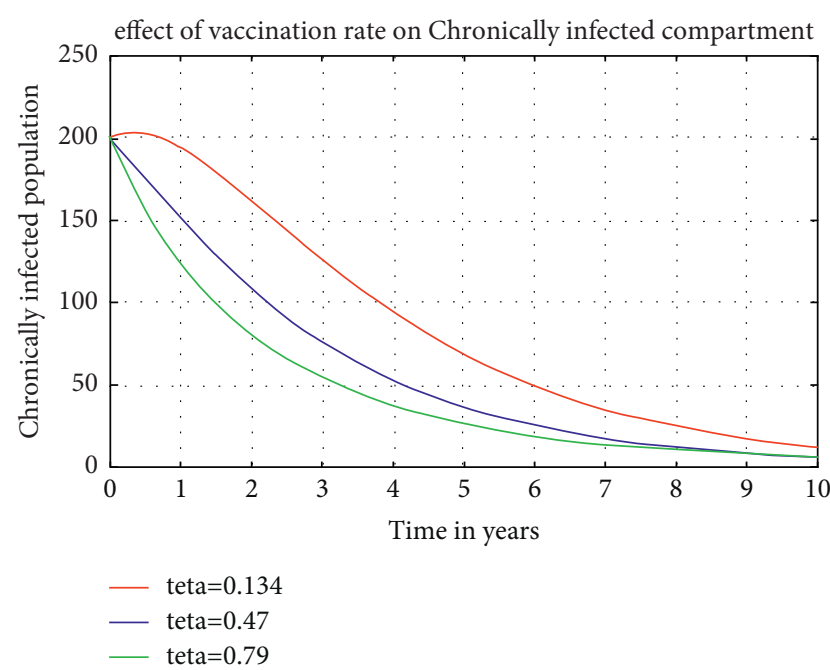

FIGURE 14: Effect of vaccination rate on chronically infected compartment.

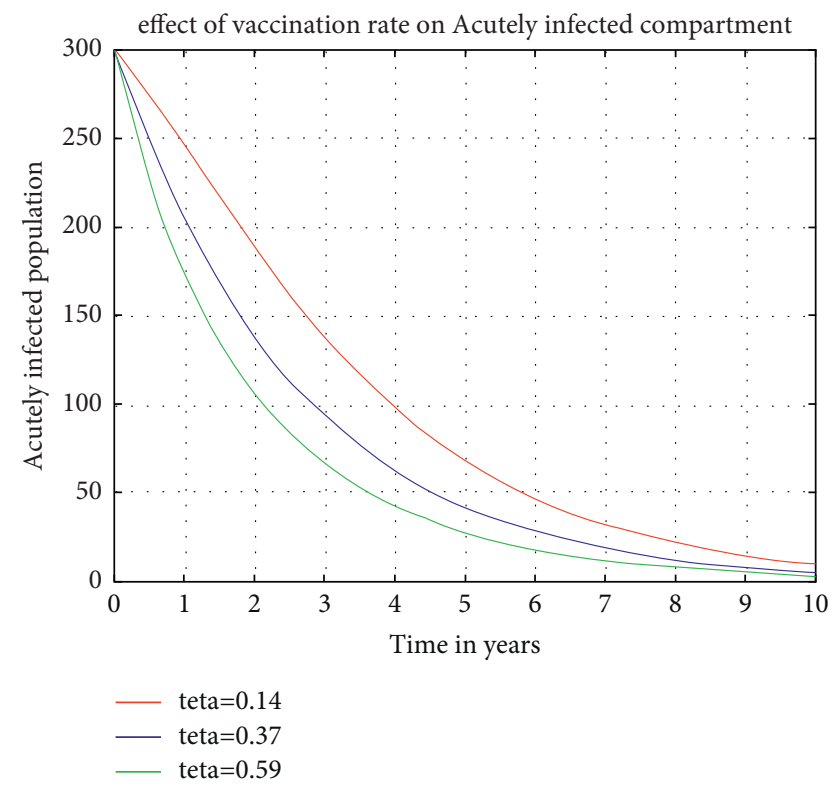

Figure 15: Effect of vaccination rate on acutely infected compartment.

\section{Conclusion}

As public health policy decisions are becoming more complex in a globalized and digitalized world, the benefits that mathematical models can offer for analyzing problems and quantifying the possible impact of interventions are huge [10]. These benefits can only be fully reaped, if mathematical modeling is sufficiently supported and facilitated within the organization of public health institutes.

In this paper, we proposed an SEACTR model of hepatitis $B$ virus infection with two controls: vaccination and treatment. First, we analyzed the dynamic behavior of the system for constant controls. In the constant controls case, we calculated the effective reproduction number and

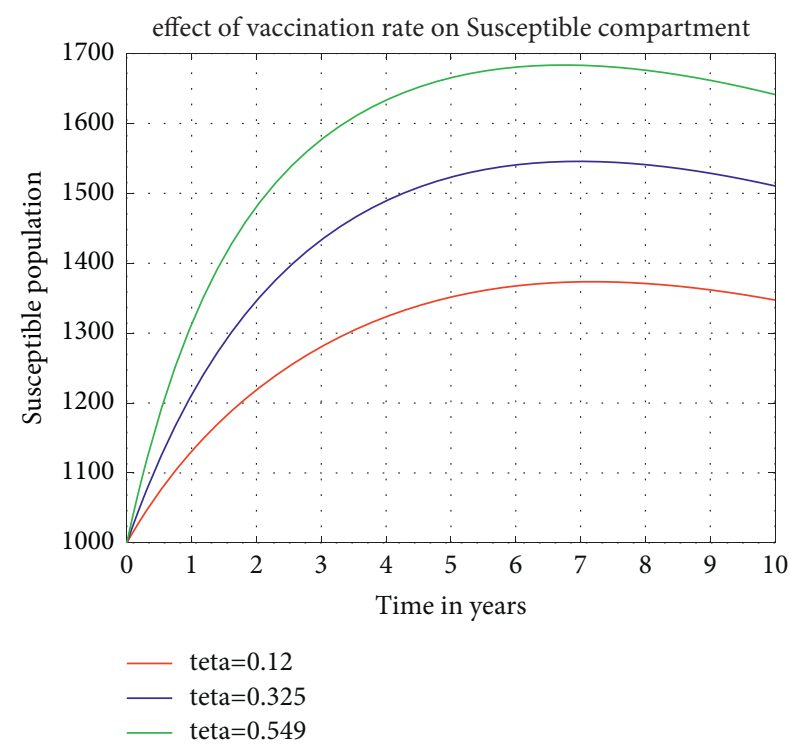

Figure 16: Effect of vaccination rate on susceptible population.

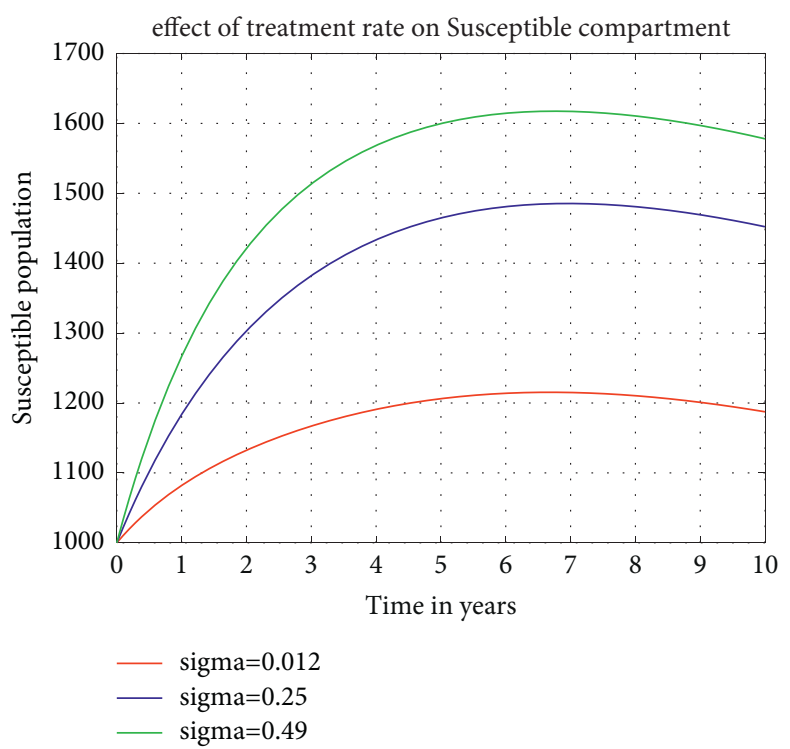

Figure 17: Effect of treatment rate on susceptible population.

investigated the existence and stability of equilibria. There are two nonnegative equilibria of the system, namely, the disease-free and endemic. We see that the disease-free equilibrium always exists and is locally asymptotically stable if $R_{\mathrm{Eff}}<1$, and endemic equilibrium exists and is locally asymptotically stable if $R_{\text {Eff }}>1$. We evaluated effective reproduction number versus exposure rate with respect to each intervention and also with respect to both vaccination and treatment interventions. Since, decreasing exposure rate decreases reproduction number of HBV disease, this shows the increment of susceptible population. By education campaign, it is possible to decrease exposure rate of the population to $\mathrm{HBV}$ disease. We also compared reproduction number as $R_{V T}<R_{V \text { Only }}<R_{T \text { Only }}<R_{0}$ showing that both vaccination and treatment interventions play a vital role in 


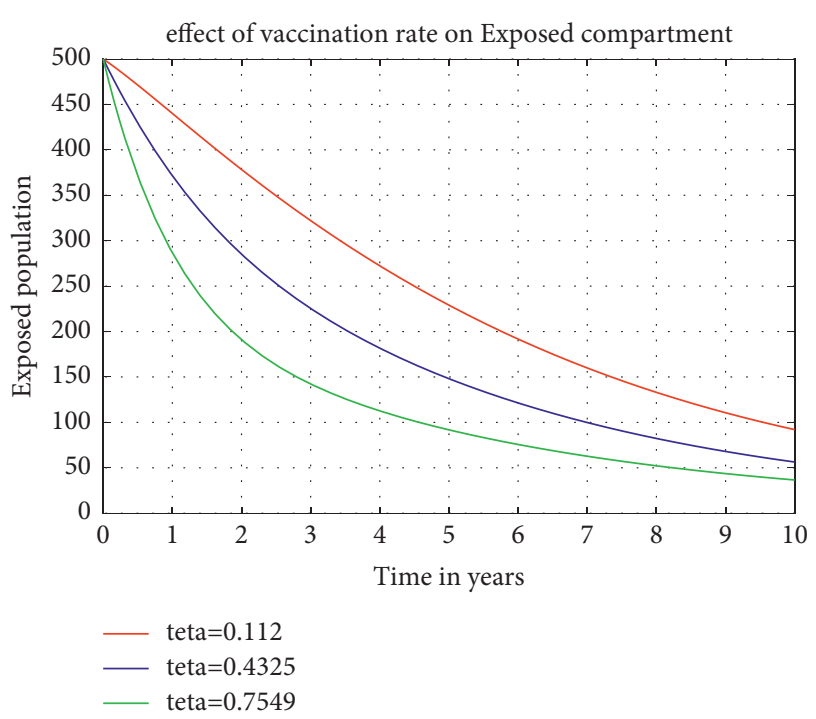

FIGURE 18: Effect of vaccination rate on exposed population.

decreasing reproduction number of $\mathrm{HBV}$ disease compared to no intervention reproduction number. The index of the sensitivity parameter shows that the parameters $c_{2}$ and $c_{1}$ have the most dominant sensitivity to raise the endemicity of $\mathrm{HBV}$. On the other hand, the parameters $\theta$ and $\sigma$ are the ones that can decrease the endemicity of $\mathrm{HBV}$ the most.

\section{Significant Outcomes of Our Work}

The efficiency of the measurement against HBV, therefore, can be assured by suppressing transfer rate from exposed class to acute and chronic infective classes and maximizing vaccination for the whole population and treatment for chronic infective population. Mass hepatitis B vaccination in the new born population largely decreases the carriage of the virus and the diseases associated with acute and chronic infection, including cirrhosis and hepatocellular carcinoma.

Worldwide, about 360 million people are chronically infected with the virus and they continue to spread the virus to others. They themselves are at the risk of chronic liver diseases and hepatocellular carcinoma. The transmission route of HBV can now be interrupted by immunizing all the susceptible population and the infection can be treated by nucleotide analogs, antivirals, or interferons. Nevertheless, the most effective means is to immunize all susceptible individuals, especially young children, with safe and efficacious vaccines.

To begin with, the existing HBV carriers can now be treated effectively and viral load can decrease to undetectable levels, so that infection does not spread rampantly. Another important strategy is to interrupt the transmission route which can be reduced after education campaign for the public and medical personnel.

To investigate the effect of vaccination and treatment on transmission of HBV, we analyzed by numerical simulation. We have shown the most sensitive parameters of our model which can be epidemiologically controlled are the HBV acute infective class transfer rate $c_{2}$ and chronic infective class transfer rate $c_{1}$. So, it is reasonable to recommend the use of intervention strategies for $\mathrm{HBV}$ transmission in making $c_{1}$ less than 0.267 and $c_{2}$ less than 0.192 . These intervention strategies are vaccination and treatment. By making the sensitive parameters vaccination rate $\theta$ greater than 0.41 and treatment rate $\sigma$ greater than 0.682 , it is possible to decrease transmission of $\mathrm{HBV}$ in the society.

From Figure 7, we see that the number of susceptible populations decreases than when there is no control because reproduction number of $\mathrm{HBV}$ is greater in the absence of control. In this case, most of this population tends to the infected class. Again, when only treatment control is applied, then the number of susceptible populations is not much different than the population in the case having no control. But the susceptible population differs much from these two strategies if we apply the strategies of only vaccination control and both vaccination and treatment controls make reproduction number of the disease decrease fast. At a high rate of vaccination, the sensitive population density is reduced to a very low level initially and then it takes longer time to restore the steady-state value.

\section{Data Availability}

The authors used secondary data from related literatures published on the HBV transmission dynamics case and cited the source of their data in the references part $[12,20,23,32,33]$.

\section{Conflicts of Interest}

The authors declare that they have no conflicts of interest.

\section{References}

[1] CDC, "Hepatitis B virus: a comprehensive strategy for eliminating transmission in the United States through universal childhood vaccination," Recommendations of the Immunization Practices Advisory Committee (ACIP)," MMWR Recommendations and Reports, vol. 40, no. RR-13, pp. 1-25, 1991.

[2] J. Mann and M. Roberts, "Modelling the epidemiology of hepatitis B in New Zealand," Journal of Theoretical Biology, vol. 269, no. 1, pp. 266-272, 2011.

[3] M. K. Libbus and L. M. Phillips, "Public health management of perinatal hepatitis B virus," Public Health Nursing, vol. 26, no. 4, pp. 353-361, 2009.

[4] N. C. Grassly and C. Fraser, "Mathematical models of infectious disease transmission," Nature Reviews Microbiology, vol. 6, no. 6, pp. 477-487, 2008.

[5] G. M. Knight, N. J. Dharan, G. J. Fox et al., "Bridging the gap between evidence and policy for infectious diseases: how models can aid public health decision-making," International Journal of Infectious Diseases, vol. 42, pp. 17-23, 2016.

[6] WHO and B Hepatitis, Fact Sheet No. 204, The World Health Organization, Geneva, Switzerland, 2000, http://www.who. int/mediacentre/factsheets/fs204/en.

[7] G. Zaman, Y. Han Kang, and I. H. Jung, "Stability analysis and optimal vaccination of an SIR epidemic model," Biosystems, vol. 93, no. 3, pp. 240-249, 2008.

[8] L Star and SM Moghadas, "The role of mathematical modelling in public health planning and decision making," Purple 
Paper, National Collaborative Center for Infectious Diseases, Winnipeg, Manitoba, 2010.

[9] A. Camacho, R. M. Eggo, N. Goeyvaerts et al., "Real-time dynamic modelling for the design of a cluster-randomized phase 3 Ebola vaccine trial in Sierra Leone," Vaccine, vol. 35, no. 4, pp. 544-551, 2017.

[10] J. P. Chretien, S. Riley, and D. B. George, "Mathematical modeling of the West Africa Ebola epidemic," Elife, 2015.

[11] H. C. Boshuizen, S. K. Lhachimi, P. H. M. Van Baal et al., "The DYNAMO-HIA model: an efficient implementation of a risk factor/chronic disease Markov model for use in Health Impact Assessment (HIA)," Demography, vol. 49, no. 4, pp. 1259-1283, 2012.

[12] H. C. Boshuizen, M. J. J. C. Poos, M. Van Den Akker et al., "Estimating incidence and prevalence rates of chronic diseases using disease modeling," Population Health Metrics, vol. 15 , no. 1 , p. 13, 2017.

[13] R. Fehr, F. Hurley, O. C. Mekel, and J. P. Mackenbach, "Quantitative health impact assessment: taking stock and moving forward: table 1," Journal of Epidemiology \& Community Health, vol. 66, no. 12, pp. 1088-1091, 2012.

[14] R. T. Hoogenveen, P. H. M. Van Baal, and H. C. Boshuizen, "Chronic disease projections in heterogeneous ageing populations: approximating multi-state models of joint distributions by modelling marginal distributions," Mathematical Medicine and Biology, vol. 27, no. 1, pp. 1-19, 2010.

[15] P. Van Den Driessche and J. Watmough, "Reproduction numbers and sub-threshold endemic equilibria for compartmental models of disease transmission," Mathematical Biosciences, vol. 180, no. 1-2, pp. 29-48, 2002.

[16] S. Zhao, Z. Xu, and Y. Lu, "A mathematical model of hepatitis $B$ virus transmission and its application for vaccination strategy in China," International Journal of Epidemiology, vol. 29, no. 4, pp. 744-752, 2000.

[17] K. M. Owolabi, "Modeling the mechanics of viral kinetics under immune control during primary infection of HIV-1 with treatment in fractional order," Physica A, vol. 545, p. 123816, 2020.

[18] M. Furman, "Numerical treatment of a nonlinear dynamical Hepatitis-B model: an evolutionary approach," European Physical Journal Plus, vol. 135, no. 12, p. 941, 2020.

[19] N. Ahmad, "Modeling the transmission dynamics of COVID19 pandemic in Caputo type fractional derivative," Journal of Multiscale Modelling, vol. 12, no. 3, p. 2150006, 2021.

[20] P. Ahmad, "Chaotic dynamics of a fractional order HIV-1 model involving AIDS-related cancer cells," Chaos, Solitons \& Fractals, vol. 140, p. 110272, 2020.

[21] H. Houweling, M. Verweij, and E. J. Ruitenberg, "Criteria for inclusion of vaccinations in public programmes," Vaccine, vol. 28 , no. 17, pp. 2924-2931, 2010.

[22] AV Kamyad, R Akbari, AA Heydari, and A Heydari, "Mathematical modeling of transmission dynamics and optimal control of vaccination and treatment for hepatitis B virus," Computational and Mathematical Methods in Medicine, vol. 201415 pages, Article ID 475451, 2014.

[23] G. F. Medley, N. A. Lindop, W. J. Edmunds, and D. J. Nokes, "Hepatitis-B virus endemicity: heterogeneity, catastrophic dynamics and control," Nature Medicine, vol. 7, no. 5, pp. 619-624, 2001.

[24] S. Thornley, C. Bullen, and M. Roberts, "Hepatitis B in a high prevalence New Zealand population: a mathematical model applied to infection control policy," Journal of Theoretical Biology, vol. 254, no. 3, pp. 599-603, 2008.
[25] L. Zou, W. Zhang, and S. Ruan, "Modeling the transmission dynamics and control of hepatitis B virus in China," Journal of Theoretical Biology, vol. 262, no. 2, pp. 330-338, 2010.

[26] T. Zhang, K. Wang, and X. Zhang, Modeling and analyzing the transmission dynamics of HBV epidemic in Xinjiang, PLoS One, China, Article ID e0138765, 2015.

[27] J. Pang, J.-A. Cui, and X. Zhou, "Dynamical behavior of a hepatitis B virus transmission model with vaccination," Journal of Theoretical Biology, vol. 265, no. 4, pp. 572-578, 2010.

[28] C. J. E. Metcalf, W. J. Edmunds, and J. Lessler, "Six challenges in modelling for public health policy," Epidemics, vol. 10, pp. 93-96, 2015.

[29] O. Diekmann, J. A. Heesterbeek, and J. A. Metz, "On the definition and the computation of the basic reproduction ratio $\mathrm{R} 0$ in models for infectious diseases in heterogeneous populations," Journal of Mathematical Biology, vol. 28, no. 4, pp. 365-382, 1990.

[30] J. P. Lasalle, The Stability of Dynamical Systems, SIAM, Philadelphia, PA, USA, 1976.

[31] G. T. Tilahun, "Modeling co-dynamics of pneumonia and meningitis diseases," Advances in Difference Equations, vol. 2019, no. 1, Article ID 2087, 2019.

[32] G. Birkhoff and G.-C. Rota, Ordinary Differential Equations, John Wiley \& Sons, New York, NY, USA, 4th edition, 1989.

[33] A. Pandey, K. E. Atkins, J. Medlock et al., "Strategies for containing Ebola in West Africa," Science, vol. 346, no. 6212, pp. 991-995, 2014. 